\title{
High-spectral resolution $M$-band observations of CO Rot-Vib absorption lines towards the Galactic center ${ }^{\star}$
}

\author{
J. Moultaka ${ }^{1}$, A. Eckart ${ }^{2,3}$, K. Tikare ${ }^{4}$, and A. Bajat ${ }^{5}$ \\ ${ }^{1}$ IRAP, Université de Toulouse, CNRS, CNES, UPS, 14 avenue Edouard Belin, 31400 Toulouse, France \\ e-mail: jihane.moultaka@irap.omp.eu \\ 2 I Physikalisches Institut, Universität zu Köln, Zülpicher Str. 77, 50937 Köln, Germany \\ ${ }^{3}$ Max-Planck-Institut für Radioastronomie, Auf dem Hügel 69, 53121 Bonn, Germany \\ ${ }^{4}$ Lulea University of Technology, Kiruna, Sweden \\ ${ }^{5}$ Institute of Physics of the Czech Academy of Sciences, Prague, Czech Republic
}

Received 22 January 2019 / Accepted 22 April 2019

\begin{abstract}
Context. In the near- to mid-infrared wavelength domain, bright continuum sources in the central parsec of the Galactic center (GC) are subject to foreground absorption. These sources therefore represent ideal probes of the intervening material that is responsible for the absorption along the line of sight.

Aims. Our aim is to shed light on the location and physics of the absorbing clouds. We try to find out which of the gaseous absorbing materials is intimately associated with the GC and which one is associated with clouds at a much larger distance.

Methods. We used the capabilities of CRIRES spectrograph located at ESO Very Large Telescope in Chile to obtain absorption spectra of individual lines at a high spectral resolution of $R=50000$, that is, $5 \mathrm{~km} \mathrm{~s}^{-1}$. We observed the ${ }^{12} \mathrm{CO} \mathrm{R}(0), \mathrm{P}(1), \mathrm{P}(2), \mathrm{P}(3), \mathrm{P}(4), \mathrm{P}(5)$, $\mathrm{P}(6), \mathrm{P}(7)$ and $\mathrm{P}(9)$ transition lines, applied standard data reduction, and compared the results with literature data.

Results. We present the results of CRIRES observations of 13 infrared sources located in the central parsec of the Galaxy. The data provide direct evidence for a complex structure of the interstellar medium along the line of sight and in the close environment of the central sources. In particular we find four cold foreground clouds at radial velocities $v_{\text {LSR }}$ of the order of $-145,-85,-60$, and $-40 \pm 15 \mathrm{~km} \mathrm{~s}^{-1}$ that show absorption in the lower transition lines from $\mathrm{R}(0)$ to $\mathrm{P}(2)$ and in all the observed spectra. We also find in all sources an absorption in velocity range of $50-60 \mathrm{~km} \mathrm{~s}^{-1}$, possibly associated with the so-called $50 \mathrm{~km} \mathrm{~s}^{-1}$ cloud and suggesting an extension of this cloud in front of the GC. Finally, we detect individual absorption lines that are probably associated with material much closer to the center and with the sources themselves, suggesting the presence of cold gas in the local region.
\end{abstract}

Key words. Galaxy: center - galaxies: nuclei - infrared: ISM

\section{Introduction}

The central parsec of our Galaxy, often referred to as the Galactic center (GC), is a very intriguing region that has been studied for decades. It harbors a supermassive black hole, Sgr $\mathrm{A}^{\star}$ (Schödel et al. 2003; Ghez 2003), surrounded by a dense central cluster where late-type red giants, young massive stars, and bowshock shaped infrared (IR) sources are identified (Ott et al. 1999; Paumard et al. 2004; Perger et al. 2008; Tanner et al. 2003; Eckart et al. 2004). The interstellar medium (ISM) in the region of the GC consists of several components: in the very central parsec, it shows a spiral feature called "the minispiral" (or Sgr A West) composed of dust and ionized gas where thin dust filaments are also observed, probably resulting from interactions with stellar winds (e.g., Muzic et al. 2007; Paumard et al. 2001). Furthermore, the GC is surrounded by the circumnuclear disk (CND), a molecular ring of dense gas and dust (Guesten et al. 1987; Garcia-Marin et al. 2011; Mossoux et al. 2018). These structures are responsible in part for the very high extinction observed towards the GC (about 35 mag in the visible; e.g., Schödel et al. 2007; Scoville et al. 2003). The main obscuration however is due to the diffuse ISM and dense molecular

\footnotetext{
* The reduced spectra are only available at the CDS via anonymous ftp to cdsarc.u-strasbg. fr (130.79.128.5) or via http://cdsarc. u-strasbg.fr/viz-bin/qcat?]/A+A/626/A44
}

clouds present along the line of sight (LOS). Only one third of it would be due to foreground dense molecular clouds (Whittet et al. 1997). Also, in our previous works (Moultaka et al. 2004, 2005, 2015a) we showed that local absorptions probably occur in the direct environment of the GC IR sources. As a matter of fact, the spectra of luminous IR sources in the $L$-band (from 2.7 to $4.2 \mu \mathrm{m}$ ) revealed absorption features due to water ices and hydrocarbons that we claimed to be present in the local medium of the central parsec. These absorptions were attributed earlier by other authors to material in the foreground molecular clouds (e.g., Chiar et al. 2000, 2002).

In Fig. 1, we show the complex observational situation with respect to absorption lines that occur along the LOS towards the GC. The absorptions can occur at several locations, some of which may be associated with the immediate environment of the stellar cluster and the ISM structure close to or within it.

More recently, we used the ISAAC ESO/VLT spectrograph capabilities in the low-resolution $(R=800)$ mode of the $M$-band wavelength range (from 4.4 to $5.1 \mu \mathrm{m}$ ) to study the spectra of 15 bright GC sources (Moultaka et al. 2009) and to map a significant region of the central parsec in the $M$-band (Moultaka et al. 2015b). This resulted in the high-quality $M$-band spectra $\left(<0.8^{\prime \prime}\right.$ resolution and high signal-to-noise ratio $\left.(\mathrm{S} / \mathrm{N})\right)$ and in a larger number of GC sources than previously published works (Lacy et al. 1984; Tegler et al. 1993; Geballe et al. 1986, 1989; 


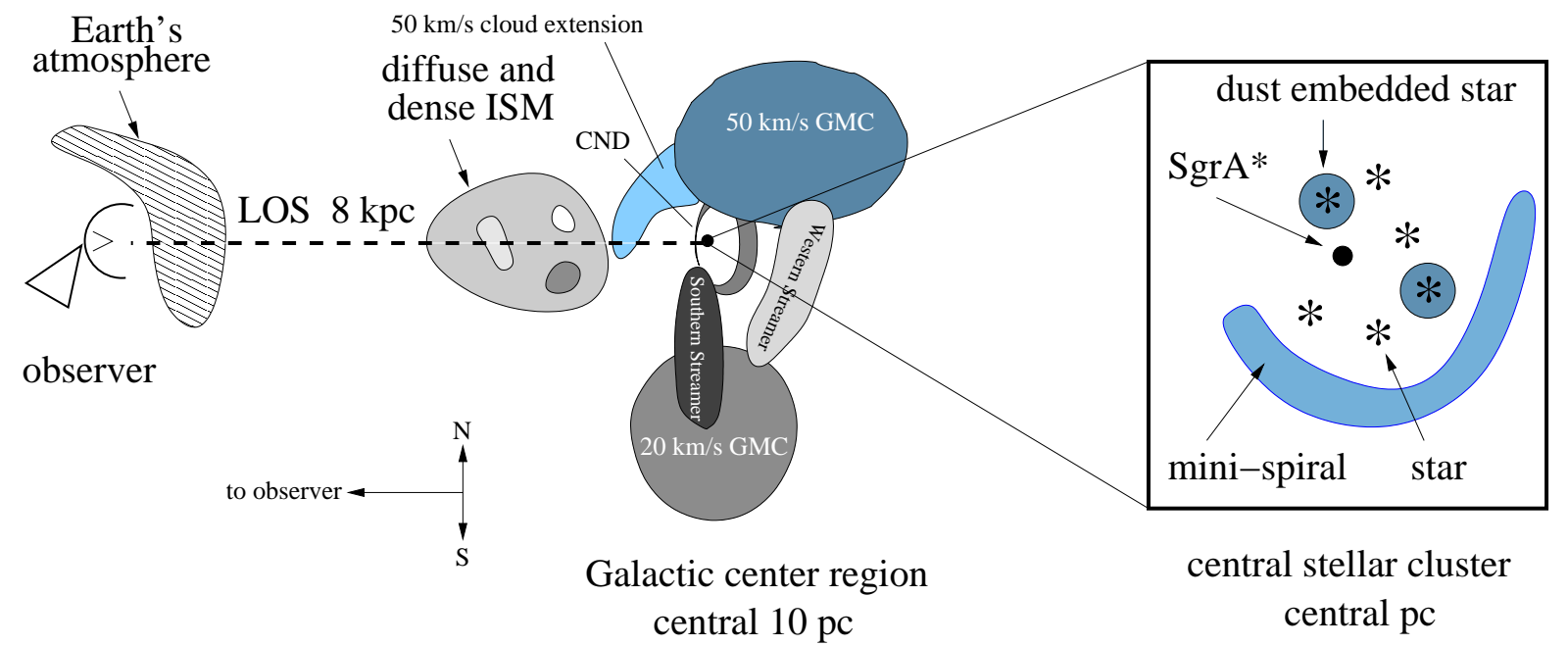

Fig. 1. Schematic view explaining the origin of absorption lines towards the GC. Part of the absorption is taking place in the Earth's atmosphere (left). This is usually calibrated using reference stars. However, residuals may still be present or genuine astrophysical absorption lines may be difficult to access if they fall onto an atmospheric line. Part of the absorption may take place in the cold, dense, and diffuse ISM in the LOS towards the GC (middle, Whittet et al. 1997). Most of this absorption probably takes place within the final few hundred parsecs. Goto et al. (2014) point out that the absorptions cover a wide range of velocities and LOS locations within the central molecular zone close to the central stellar cluster. Within the central $10 \mathrm{pc}$ there are a few molecular cloud complexes that come quite close to the LOS (right). Following Lee et al. (2008) some of them are indicated here. The $50 \mathrm{~km} \mathrm{~s}^{-1}$ cloud has been indicated in light blue. See comments on the $50 \mathrm{~km} \mathrm{~s}^{-1}$ extension in the caption of Fig. 6 . Finally, individual absorptions in the local environment of the central cluster may take place (right inset). This may happen against the minispiral or against the bowshocks, shells, and circumstellar envelopes of some or the MIR excess sources in the central cluster. Some representative structures have been indicated in the inset.

McFadzean et al. 1989; Moneti et al. 2001). Moreover, the fundamental $\mathrm{P}$ and $\mathrm{R}$ branches of the ro-vibrational transition lines of the ${ }^{12} \mathrm{CO}$ and ${ }^{13} \mathrm{CO}$ gas-phases (around 4.666 and $4.77 \mu \mathrm{m}$, respectively) were resolved in the GC. We found that an appreciable amount of the CO in the foreground ISM may also originate from solid phase, stuck to the surface of dust grains, and that both gas and dust of the diffuse medium are responsible for a major part of the extinction. We performed a first-order correction of individual GC source spectra for the foreground absorption due to $\mathrm{CO}$-ice and gas. The resulting corrected spectra for the $\mathrm{CO}$-ice absorption at $4.675 \mu \mathrm{m}$ revealed residual absorption in a number of sources. The residual absorption may be due to variations in the foreground LOS extinction. In general, all previous works concluded that it is unlikely that the molecular clouds are close to the GC because the $\mathrm{CO}$ ice is unlikely to survive temperatures higher than $20 \mathrm{~K}$ unless it is located in an environment of water ice and other molecules (e.g., McFadzean et al. 1989). We claimed that part of the residual $\mathrm{CO}$-ice may be due to GC material (i.e., minispiral) or to material within the circumstellar shells or bow shocks of the sources. We showed that given the structure of the GC and the presence of water ices (Moultaka et al. 2004, 2005, 2015a), bowschock sources, and dust filaments, CO-ice may survive in this environment. Moreover, we observed a residual CO-gas absorption in the corrected spectra. Assuming that the residual gas material is located in the minispiral or in the circumstellar shells of the dust-embedded bow-shock sources, we obtained gas masses of the circumstellar shells of the order of $10^{-3} M_{\odot}$ in agreement with an independent estimate of circumstellar shell masses using published CO-(7-6) and FIR[OI] data. Consequently, we deduced that a substantial part of the $4.666 \mu \mathrm{m}$ gaseous $\mathrm{CO}$ absorption could be due to intrinsic circumstellar material, although Geballe et al. (1989) attributed the CO R(2) and R(5) lines towards IRS 1, 2, 3, 5, 6, 7, and 8 GC sources observed at velocities between 0 and $75 \mathrm{~km} \mathrm{~s}^{-1}$ to the gas in the spiral arms along the LOS to the GC and to the 20 and $50 \mathrm{~km} \mathrm{~s}^{-1}$ known foreground clouds close to the GC. If this is the case and if our conclusion/assumption was correct, the $\mathrm{CO}$ absorptions would occur at the radial velocities of the sources, many of which are larger than the 20 and $50 \mathrm{~km} \mathrm{~s}^{-1}$ cloud velocities (see Table 1).

In this paper, we present the results of our spectroscopic observations $^{1}$ of individual IR sources of the central parsec at a higher spectral resolution $\left(R=50000\right.$ i.e., $\left.5 \mathrm{~km} \mathrm{~s}^{-1}\right)$ than what we have previously accomplished, using the capabilities of CRIRES spectrograph located at ESO Very Large Telescope. Our goal is to identify intrinsic gaseous $\mathrm{CO}$ absorption by checking if the $\mathrm{CO}$ lines occur at the radial velocities of the sources and consequently if the $\mathrm{CO}$ is located in the circumstellar medium of these sources. In the following section, we describe the observation setups and the data-reduction steps. In Sect. 3, we show and discuss our results in terms of local and foreground absorptions and finally we conclude in the last section.

\section{Observations and data reduction}

The observations were obtained during period $93^{2}$ on three half nights (18, 19, and 20 May, 2014) with CRIRES high-resolution spectrograph located at UT1 of the VLT/ESO observatory. We used a $0.4^{\prime \prime}$ slit in the high-resolution mode of CRIRES which results in a spectral resolution of about 50000 .

For our scientific goals, we observed at two reference wavelengths, 47233 and $47363 \AA$, according to the definition of the CRIRES manual (i.e., the reference wavelength is the one at pixel 512 of the third detector). At each of the reference wavelengths, the observations were obtained in four distinct ranges that do not overlap, corresponding to the four detectors of the instrument. In Table 2 we show the obtained ranges for each reference wavelength. On average, the exposure time was about $1.5 \mathrm{~h}$ per slit position and instrument setup. In total, six slit

\footnotetext{
1 Based on observations collected at the European Southern Observatory under ESO programme 093.C-0086(A).

2 Observing program run 093.C-0086(A).
} 
Table 1. Radial velocities of the individual observed sources (in Col. 3) as found in the literature (references are given in Col. 4).

\begin{tabular}{|c|c|c|c|c|}
\hline Source & Type & $\begin{array}{l}\text { Radial velocity } \\
\qquad\left(\mathrm{km} \mathrm{s}^{-1}\right)\end{array}$ & References & $\begin{array}{l}v_{\text {LSR }} \text { of a local absorption } \\
\left(\mathrm{km} \mathrm{s}^{-1}\right)\end{array}$ \\
\hline IRS $1 \mathrm{~W}$ & Red & $35 \pm 20$ & Paumard et al. (2006) & $\begin{array}{l}35 \pm 15 \text { in } \mathrm{R}(0) \\
50 \pm 15 \text { in } \mathrm{P}(1)\end{array}$ \\
\hline IRS 2S & Cool & $107 \pm 20$ & Genzel et al. (2000) & $-110 \pm 15$ in $\mathrm{R}(0) \mathrm{P}(1)$ and $\mathrm{P}(2)$ \\
\hline IRS 2L & Red & - & & $\begin{array}{l}60 \pm 15 \text { in } \mathrm{R}(0), \mathrm{P}(1), \mathrm{P}(2) \text { and } \mathrm{P}(3) \\
-\mathbf{1 0 5} \pm \mathbf{1 5} \text { in } \mathrm{R}(0), \mathrm{P}(1) \text { and } \mathrm{P}(3)\end{array}$ \\
\hline IRS 3 & Red & - & & $60 \pm 15$ in $\mathrm{R}(0), \mathrm{P}(1), \mathrm{P}(2), \mathrm{P}(3), \mathrm{P}(4), \mathrm{P}(6)$ and $\mathrm{P}(7)$ \\
\hline IRS 5 & Cool & $110 \pm 60$ & Tanner et al. (2005) (flow) & $70 \pm 15$ in $P(1), P(2)$, and $P(3)$ \\
\hline IRS 6W & Hot & $63 \pm 3$ & Zhu et al. (2008), Herbst et al. (1993) & $\begin{array}{l}-20 \pm 15 \text { in } \mathrm{P}(1), \mathrm{P}(2), \mathrm{P}(4) \text { and } \mathrm{P}(5) \\
65 \pm 15 \text { in } \mathrm{P}(1), \mathrm{P}(2), \mathrm{P}(4) \text { and } \mathrm{P}(5)\end{array}$ \\
\hline IRS $10 \mathrm{~W}$ & Cool & $80 \pm 60$ & Tanner et al. (2005) (source) & $\begin{array}{c}50 \pm 15 \text { in } \mathrm{P}(1) \text { and } \mathrm{P}(2) \\
-105 \pm 15 \text { in } \mathrm{R}(0), \mathrm{P}(1), \mathrm{P}(2) \text { and } \mathrm{P}(3)\end{array}$ \\
\hline & Cool & $195 \pm 150$ & Tanner et al. (2005) (flow) & $\begin{array}{l}110 \pm 15 \text { in } \mathrm{P}(3) \\
210 \pm 15 \text { in } \mathrm{P}(3)\end{array}$ \\
\hline IRS $12 \mathrm{~N}$ & Cool & $\begin{array}{l}-69 \pm 2 \\
-61 \pm 1\end{array}$ & $\begin{array}{l}\text { Zhu et al. (2008) } \\
\text { Figer et al. (2003) }\end{array}$ & $-85 \pm 15$ in $\mathrm{R}(0), \mathrm{P}(1), \mathrm{P}(3), \mathrm{P}(4), \mathrm{P}(6), \mathrm{P}(7)$ and $\mathrm{P}(9)$ \\
\hline IRS $13 \mathrm{E}$ & Hot & $45 \pm 60$ & Genzel et al. (2000) & $60 \pm 15$ in $\mathrm{R}(0), \mathrm{P}(1), \mathrm{P}(2)$ and $\mathrm{P}(3)$ \\
\hline IRS 16C & Hot & $125 \pm 30$ & Paumard et al. (2006) & - \\
\hline IRS 16SW & Hot & $453 \pm 4$ & Martins et al. (2006) & - \\
\hline IRS 21 & Hot & $-90 \pm 20$ & Genzel et al. (2000) & $\begin{array}{c}-60 \pm 15 \text { in } \mathrm{P}(3) \\
55 \pm 15 \text { in } \mathrm{R}(0), \mathrm{P}(1), \mathrm{P}(2) \text { and } \mathrm{P}(3)\end{array}$ \\
\hline IRS 29S & $\begin{array}{l}\text { Hot } \\
\text { Hot }\end{array}$ & $\begin{array}{l}-93 \pm 20 \\
-164 \pm 2\end{array}$ & $\begin{array}{l}\text { Genzel et al. (2000) } \\
\text { Zhu et al. (2008) }\end{array}$ & $-60 \pm 15$ in $\mathrm{P}(3)$ \\
\hline
\end{tabular}

Notes. The second column shows the spectral type of the sources listed in Col. 1. Most of the radial velocities clearly lie beyond the $20-50 \mathrm{~km} \mathrm{~s}^{-1}$ cloud velocities. For sources IRS 5 and IRS 10W, Tanner et al. (2005) give separate values for the source and the corresponding minispiral flow (see e.g., velocity field given by Zhao et al. 2010). For IRS 10W, two "flow" values are given $\left(175 \pm 130\right.$ and $215 \pm 160 \mathrm{~km} \mathrm{~s}^{-1}$ ) of which we list the average in the table. Sources IRS 1W, IRS 2L, and IRS 3 are enshrouded in dust and are therefore labeled as "red". For IRS 2L and IRS 3 we found no quoted velocity. For IRS 6W, we classify this source as "hot" using the information by Herbst et al. (1993) that the Br $\gamma$ line emission is present in its spectrum. The radial velocity given by Herbst et al. (1993) has not been tabulated in their paper but can be read off their Fig. 5 as $-166 \pm 30 \mathrm{~km} \mathrm{~s}^{-1}$ and may be associated with the gas flow in the minispiral bar. In Col. 5, we present the assumed local gas velocities measured in our spectra in the CO transition lines indicated. The values in bold are the first determinations of the radial velocities of IRS 2L and IRS 3 that we derive from the present work (see Sect. 4).

Table 2. Wavelength ranges (in Angström) of the spectra obtained at each of the CRIRES reference wavelengths.

\begin{tabular}{|c|c|c|}
\hline Reference wavelength & $\begin{array}{c}47233 \\
(\AA) \\
\end{array}$ & $\begin{array}{c}47363 \\
(\AA) \\
\end{array}$ \\
\hline Wavelength range in detector 1 & $46519-46761$ & $46656-46896$ \\
\hline Wavelength range in detector 2 & $46828-47061$ & $46962-47192$ \\
\hline Wavelength range in detector 3 & $47122-47344$ & $47253-47473$ \\
\hline Wavelength range in detector 4 & $47401-47613$ & 47529-47739 \\
\hline
\end{tabular}

positions (see Fig. 2) were used either with the $47233 \AA$ reference wavelength setup or the $47363 \AA$ one. In each of the slit positions a number of IR GC sources are observed. This resulted in data for 13 IR sources of the GC (in one or both reference wavelength settings): IRS $1 \mathrm{~W}, 2 \mathrm{~L}, 2 \mathrm{~S}, 3,5,6 \mathrm{~W}, 10 \mathrm{~W}, 12 \mathrm{~N}, 13$, 16C, 16SW, 21, and 29.

The observations were performed using the nodding and jittering techniques. Nodding consists of moving the telescope from one position to a second one along the direction of the slit and then moving it again to the first position. Jittering is a small offset that is applied randomly in addition to the nodding to avoid systematic errors and to correct for bad pixel detectors.

Thanks to the nodding technique, the dark current correction and sky emission removal are automatically obtained by subtracting the two offset images. Using the ESO CRIRES pipeline, the data were dark subtracted, corrected for nonlinearity, flatfielded and each couple of nodded images were combined. These steps are obtained when executing the crires-spec-jitter recipe of the ESO pipeline. At that step, the pipeline also extracts the brightest spectrum in the image and applies the wavelength calibration. In the present observations, several spectra are present in a single image since we positioned the slit along a series of IR sources of the GC (see Fig. 2). Moreover, the traces of the spectra in the detector images are curved. Thus, after making different unsuccessful tests to extract the spectra from one detector image and to straighten them using the pipeline recipe, we decided to use our own scripts, written with the IRAF facility ${ }^{3}$. Our scripts allow for the extraction process to be performed after straightening the traces of the spectra in the detector images. To this end and prior to this step, we extracted the four detector images from the fit output files of the criresspec-jitter recipe and saved each of them in a one-extension FITS file. The output FITS files of the CRIRES pipeline recipe have four extensions where the four detector images are saved, respectively. The wavelength calibration function was obtained using the sky emission lines present in the observations as well

3 IRAF is written and supported by the National Optical Astronomy Observatories (NOAO) in Tucson, Arizona. NOAO is operated by the Association of Universities for Research in Astronomy (AURA), Inc. under cooperative agreement with the National Science Foundation. 


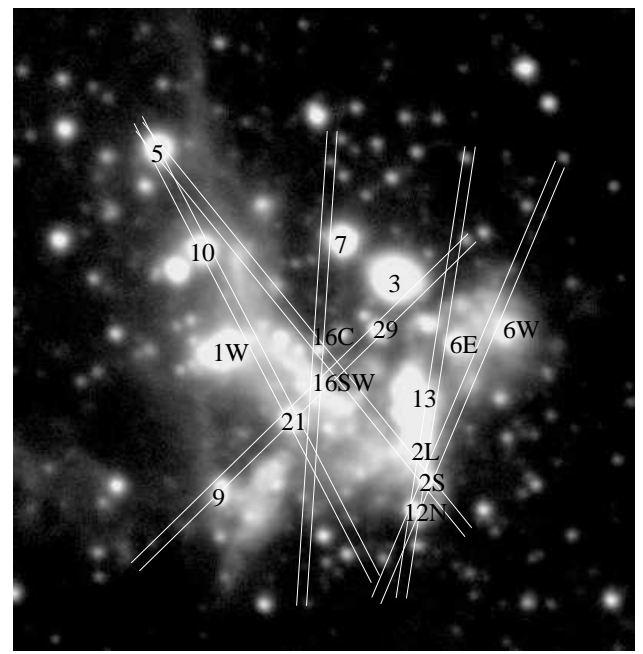

Fig. 2. Positions of the $0.4^{\prime \prime}$ slits used during the observing run. They cover the 13 observed sources listed in Table 1 . The IRS numbers of the main bright sources of the central cluster are also indicated.

as observed lines from an $\mathrm{N}_{2} \mathrm{O}$ gas-cell that was positioned in front of a halogen lamp as part of the ESO standard calibration plan. To do the wavelength calibration, we used the polynomial relation (between pixel positions and wavelengths) for each of the detector images obtained with the CRIRES ESO pipeline and applied it to the extracted spectra of each of the detector images.

The last step of the data-reduction process was the removal of telluric lines and flux calibration. This step was very difficult to perform although we observed a telluric standard soon after and before each of the science exposures using the same instrument setup and at airmasses very close to the ones obtained during the science observations. To this end, we performed several tests with the Molecfit software tool (Smette et al. 2015 and Kausch et al. 2015) dedicated to the correction of telluric absorption lines using synthetic atmospheric spectra. Most of the results were not convincing, and therefore for most of our observed spectra we performed a telluric correction by hand using our IRAF scripts and the observed telluric standard spectra. The observed telluric stars are the early-type A0V stars HR 6070, HIP 117089, and B9IV star HR 6494. The final spectra, shown in black in Figs. A.1-A.13 are not ideally corrected, as can be seen in the figures, but they are the best results that we were able to obtain. Therefore, in each of the figures presenting a science reduced spectrum, we also show the corresponding telluric star spectrum to assess our discussion and results. In general, all the wavelength ranges lying on a region of a telluric line are left out of the discussion.

\section{Results}

As explained in the previous section, most of our spectra show residual telluric lines that were very difficult to remove during the data-reduction process. In the following, all the results we present concern ${ }^{12} \mathrm{CO}$ lines in spectra for which we are confident that the features are real, that is, not telluric. In Figs. A.1-A.13 one can see examples of observed reduced spectra with the corresponding observed telluric standard stars shown in red. Thereby, we do not discuss the lines in the science spectra that fall at the location or close to the location of the telluric lines. One has to be aware that if we do not mention the presence of a line at a given velocity in a spectrum, this does not necessarily

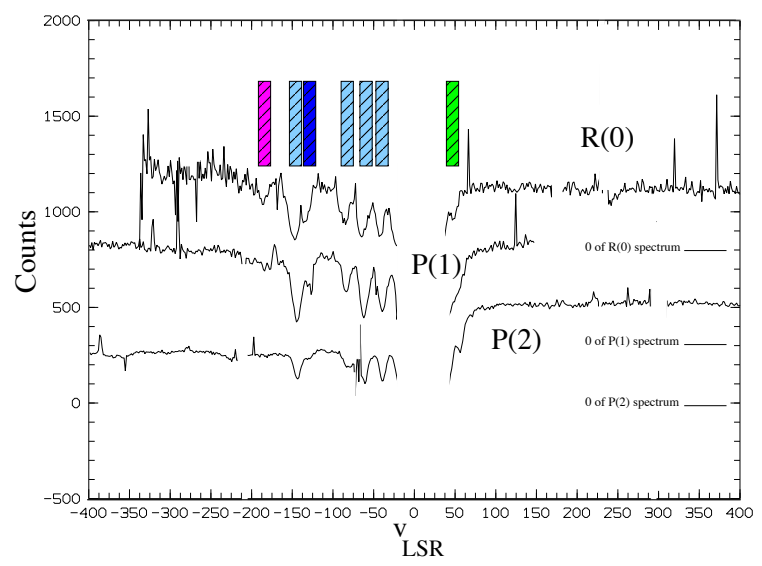

Fig. 3. Spectra of the ${ }^{12} \mathrm{CO} R(0), \mathrm{P}(1)$, and $\mathrm{P}(2)$ transition lines resulting from the addition of all the observed IR source spectra. The light blue rectangles show the positions of the lines at $v_{\mathrm{LSR}} \sim-40,-60,-85$, and $-145 \mathrm{~km} \mathrm{~s}^{-1}$ present in all the spectra and that we attribute to foreground clouds. The magenta, blue, and green rectangles show the positions of the lines at velocities $v_{\mathrm{LSR}} \sim-185,-130$, and $+50 \mathrm{~km} \mathrm{~s}^{-1}$, respectively. They are present in two of the transition line spectra $(\mathrm{R}(0)$ and $\mathrm{P}(1)$ in the case of the lines at $v_{\mathrm{LSR}} \sim-130$ and $-185 \mathrm{~km} \mathrm{~s}^{-1}$, and $\mathrm{R}(0)$ and $\mathrm{P}(2)$ in the case of the line at $v_{\mathrm{LSR}} \sim 50 \mathrm{~km} \mathrm{~s}^{-1}$ ).

mean that there is no line but it could be that at that velocity, a residual telluric line is present, meaning that we cannot provide any conclusion on its presence in the observed science spectrum. Moreover, in the following discussion, it is important to note that the absorption lines at low transitions, like $\mathrm{R}(0)$, $\mathrm{P}(1)$, and $\mathrm{P}(2)$ for example, are indicative of a low temperature in the medium where they are produced. On the contrary, high-absorption transition lines indicate higher temperatures of the medium. Therefore, the absorption lines in $\mathrm{P}(3)-\mathrm{P}(7)$ that we observe could be associated with the very local medium surrounding the individual sources.

Finally, we also note that R-branch, low $\mathrm{J}$ lines of ${ }^{13} \mathrm{CO}$ are also present in the wavelength intervals observed here but these absorptions do not contaminate any of the velocity profiles of the ${ }^{12} \mathrm{CO} \mathrm{R}-$ or P-branch lines that we present.

\subsection{Absorption along the line of sight}

The individual spectra of the observed IR sources have different S/Ns depending on the integration time and brightness of the sources. We identified a set of ${ }^{12} \mathrm{CO}$ lines, especially in $\mathrm{R}(0)$, $\mathrm{P}(1)$, and $\mathrm{P}(2)$ transitions, located at LSR velocities $\left(v_{\mathrm{LSR}}\right)$ of approximately $-40,-60,-85$, and $-145 \mathrm{~km} \mathrm{~s}^{-1}$ and another set of lines at -130 and $-185 \mathrm{~km} \mathrm{~s}^{-1}$. They are present in all or most of the spectra of the infrared sources (IRS) and are not (or are barely) contaminated by telluric lines (see Figs. A.1A.13). In order to highlight this result, we summed up all the IRS spectra for each of the ${ }^{12} \mathrm{CO} \mathrm{R}(0), \mathrm{P}(1)$, and $\mathrm{P}(2)$ line transitions and obtained the summed spectra shown in Fig. 3. We also show, in Fig. 4, the resulting spectrum obtained by adding the three previous spectra of all sources. In both figures, we distinguish the four negative velocities at $v_{\mathrm{LSR}} \sim-40,-60,-85$, and $-145 \mathrm{~km} \mathrm{~s}^{-1}$. These lines are most probably the signature of clouds located along the LOS. In the spectrum of the summed $\mathrm{R}(0)$ transition and that of the $\mathrm{P}(1)$ transition, we also distinguish two lines at $v_{\mathrm{LSR}} \sim-130$ and $-185 \mathrm{~km} \mathrm{~s}^{-1}$ that could be attributed to two other foreground clouds. Moreover, in the $R(0)$ and $\mathrm{P}(2)$ transition summed spectra we find a faint absorption at around $50 \mathrm{~km} \mathrm{~s}^{-1}$, possibly arising from the $50 \mathrm{~km} \mathrm{~s}^{-1}$ cloud at 


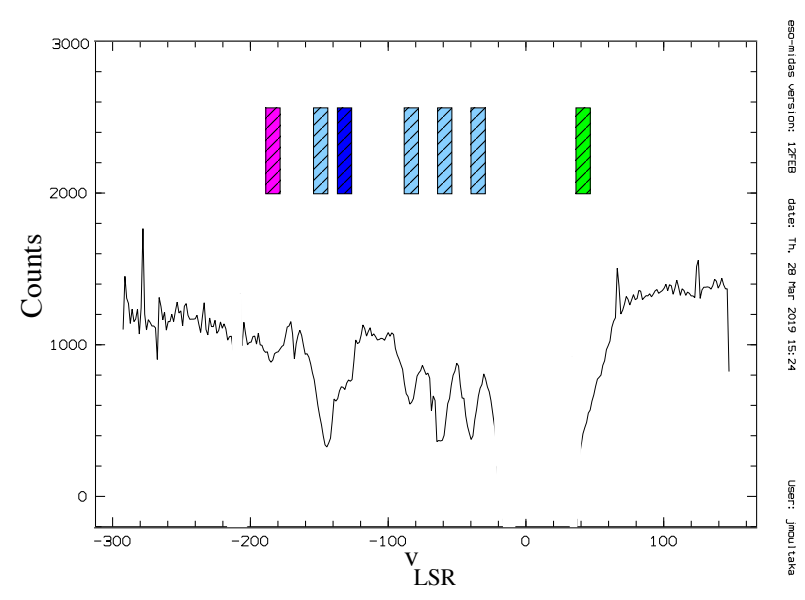

Fig. 4. Resulting spectrum of all the summed observed GC IR source spectra in the ${ }^{12} \mathrm{CO} \mathrm{R}(0), \mathrm{P}(1)$, and $\mathrm{P}(2)$ line transitions (i.e., spectrum resulting from the three spectra shown in Fig. 3). The colored rectangles have the same indications as in Fig. 3.

the GC; see, for example, Wright et al. (2001) and Uehara et al. (2017).

Finally, by comparing the velocities at the line peaks in the summed spectra between themselves and between the individual spectra, we estimate the error on our derived velocities to be of the order of $10-15 \mathrm{~km} \mathrm{~s}^{-1}$.

In the following we describe the detections of the different lines that we assume to be absorbed in clouds located along the LOS but at different distances to the GC. The association of molecular absorption with individual sources of similar radial velocity may often be explained as absorption in a bow-shock of swept up local material (e.g., IRS 1W or IRS 5). Absorption feature originating in large-scale extended shells or outflows are less likely to be expected in the harsh dynamical environment of the central stellar cluster - as these structures will be rapidly tidally destroyed. A source of some of the high-negative-velocity absorption components (between -100 and $-200 \mathrm{~km} \mathrm{~s}^{-1}$ ) could be due to $\mathrm{CO}$ in the expanding molecular ring or shell reported by Kaifu et al. (1972), Scoville (1972) and Sofue (1995). Others, for example Oka et al. (2005), attribute the high-negativevelocity absorption they observe in $\mathrm{H}_{3}^{+}$and the $\mathrm{CO}$ overtone band toward GCS3-2 to the ring. A summary of the absorption line detections is given in Table 3 and all spectra are shown in Figs. A.1-A.13.

\subsection{The case of IRS $1 W$ and IRS 3}

In Goto et al. (2014), the authors find absorptions of the ${ }^{13} \mathrm{CO}$ $v=1-0 \mathrm{P}(1)$ and the $H_{3}^{+} R(1,1)^{l}$ transitions in the spectra of IRS $1 \mathrm{~W}$ and IRS 3 , at $v_{\mathrm{LSR}}$ of $-53,-32$, and $0 \mathrm{~km} \mathrm{~s}^{-1}$. They interpret the two absorbed features at -53 and $-32 \mathrm{~km} \mathrm{~s}^{-1}$ as being produced in cold and dense gas of two lateral arms at 3 and $4 \mathrm{kpc}$ moving at these velocities. These velocities were also observed towards the GC by Moneti et al. (2001) and in radio by Sutton et al. (1990) and Serabyn \& Guesten (1986). Their finding is in agreement with the foreground velocities at $-60 \pm 15$ and $-40 \pm 15 \mathrm{~km} \mathrm{~s}^{-1}$ that we observe in our summed spectra. We note that the systematic shift of $10 \mathrm{~km} \mathrm{~s}^{-1}$ between our measurements and those obtained in Goto et al. (2014) and the above references may be due to the wavelength calibration that was not straightforward during the data-reduction process as explained in Sect. 2. In any case, this shift is inside the error bars that we obtain on the radial velocities (see Sect. 3.1).

In the present individual spectra of IRS $1 \mathrm{~W}$ and IRS 3, we find three absorptions of the ${ }^{12} \mathrm{CO} \mathrm{R}(0)$ and $\mathrm{P}(1)$ lines at velocities of the order of $v_{\mathrm{LSR}}=-60,-80$, and $-145 \mathrm{~km} \mathrm{~s}^{-1}$ even though the spectra are not of good quality (see Figs. A.1 and A.2). An absorption at approximately $-40 \mathrm{~km} \mathrm{~s}^{-1}$ is also visible in the ${ }^{12} \mathrm{CO} \mathrm{R}(0)$ and the $\mathrm{P}(1)$ transition spectra of both IRS $1 \mathrm{~W}$ and IRS 3 (see the figures), but there is a telluric line at that position. We note that most of these lines are saturated in these spectra. The absorption at $0 \mathrm{~km} \mathrm{~s}^{-1}$ found by Goto et al. (2014) was associated by the authors with gas located in foreground spiral arms. In our spectra, it can be marginally detected at low $\mathrm{S} / \mathrm{N}$ in the ${ }^{12} \mathrm{CO} \mathrm{P}(6)$ and $\mathrm{P}(7)$ lines of IRS 3 (see Fig. A.2), but it falls together with a telluric line.

\subsection{The northern arm}

In the spectra of the observed sources in the northern arm (i.e., IRS 5, IRS 10W, and IRS 21), we distinguish the prominent foreground absorption lines at LSR velocities of $-40,-60$, -85 , and $-145 \pm 15 \mathrm{~km} \mathrm{~s}^{-1}$ and also the three absorptions at $-130,-185$, and $+50 \mathrm{~km} \mathrm{~s}^{-1}$. These detections are more specifically observed in the $\mathrm{R}(0)$ and $\mathrm{P}(1)$ transition line spectra (see Figs. A.3-A.5). The $-185 \mathrm{~km} \mathrm{~s}^{-1}$ absorption is less visible in the $\mathrm{R}(0)$ and $\mathrm{P}(1)$ spectra of IRS 21 as can be seen in Fig. A.5.

\subsection{The bar and around the minicavity}

All the observed sources that are located in the bar of the minispiral and around the minicavity, namely IRS $2 \mathrm{~S}, 2 \mathrm{~L}, 12 \mathrm{~N}, 13 \mathrm{E}$, 29S, and 6W (see Fig. 2), show the four foreground absorptions at $-40,-60,-85$, and $-145 \pm 15 \mathrm{~km} \mathrm{~s}^{-1}$ in their $\mathrm{R}(0)$, $\mathrm{P}(1)$, and/or $\mathrm{P}(2)$ transition spectra (see Figs. A.6 to A.11). The spectra also show the $50 \mathrm{~km} \mathrm{~s}^{-1}$ absorption in almost all transitions from $\mathrm{R}(0)$ to $\mathrm{P}(3)$. However, the $-185 \pm 15 \mathrm{~km} \mathrm{~s}^{-1}$ and $-130 \pm 15 \mathrm{~km} \mathrm{~s}^{-1}$ clouds are not detected in the spectra of IRS 29S and 6W, respectively.

The IRS 13 spectra in the $\mathrm{R}(0)$ and $\mathrm{P}(1)$ transitions show an additional line at about approximately $-210 \pm 15 \mathrm{~km} \mathrm{~s}^{-1}$ (see Fig. A.8). To the south of IRS 13, around the minicavity, the IRS 2L, IRS $2 \mathrm{~S}$, and IRS $12 \mathrm{~N}$ sources also show absorptions at the same velocity (i.e., approximately $-210 \pm 15 \mathrm{~km} \mathrm{~s}^{-1}$ ) in their $\mathrm{R}(0)$ (except IRS 2S) and $\mathrm{P}(1)$ transition spectra (see Figs. A.9-A.11). This indicates that the line at this velocity probably arises from material in this IRS $13-2 \mathrm{~L}-2 \mathrm{~S}-12 \mathrm{~N}$ complex around the minicavity. We assume that this material is closer to the GC than the other foreground clouds even though the line is only detected at low transitions, because its detection is confined to only four sources of the central parsec.

\subsection{The helium stars}

The $\mathrm{R}(0)$ and $\mathrm{P}(1)$ spectra of the helium stars IRS 16C and IRS 16SW show exclusively the lines that we attribute to cold dense LOS clouds with LSR velocities of $-40,-60$, -85 , and $-145 \pm 15 \mathrm{~km} \mathrm{~s}^{-1}$ as well as the $50 \pm 15 \mathrm{~km} \mathrm{~s}^{-1}$ line (see Figs. A.12 and A.13) also at higher transitions. The latter is most probably absorbed in the well-known $50 \mathrm{~km} \mathrm{~s}^{-1}$ cloud that is closer to the GC. We note that in the $\mathrm{P}(1)$ and the $\mathrm{P}(2)$ spectra of IRS $16 \mathrm{C}$, there is a telluric at the position of the approximately $-40 \mathrm{~km} \mathrm{~s}^{-1}$ line and another at the approximately $-145 \mathrm{~km} \mathrm{~s}^{-1}$ line. Moreover, in the $\mathrm{R}(0)$ spectrum of IRS 16SW, a telluric is also present close to 
Table 3. Summary of the observed absorption lines assumed to arise from foreground clouds of the GC sources listed in Col. 1.

\begin{tabular}{|c|c|c|c|c|c|c|}
\hline Source & Type & $\begin{array}{c}\text { Stellar radial } \\
\text { velocity }\left(\mathrm{km} \mathrm{s}^{-1}\right)\end{array}$ & $\begin{array}{c}\text { Lines at } v_{\mathrm{LSR}}= \\
-145,-85,-60,-40 \mathrm{~km} \mathrm{~s}^{-1} \\
\text { observed in summed } \\
\mathrm{R}(0), \mathrm{P}(1) \text { and } \mathrm{P}(2) \\
\text { spectra }\end{array}$ & $\begin{array}{c}\text { Lines at } v_{\mathrm{LSR}}= \\
-185 \mathrm{~km} \mathrm{~s}^{-1} \\
\text { observed in summed } \\
\mathrm{R}(0) \text { and } \mathrm{P}(1) \\
\text { spectra }\end{array}$ & $\begin{array}{c}\text { Lines at } v_{\mathrm{LSR}}= \\
-130 \mathrm{~km} \mathrm{~s}^{-1} \\
\text { observed in summed } \\
\mathrm{R}(0) \text { and } \mathrm{P}(1) \\
\text { spectra }\end{array}$ & $\begin{array}{c}\text { Lines at } v_{\mathrm{LSR}}= \\
50 \mathrm{~km} \mathrm{~s}^{-1} \\
\text { observed in summed } \\
\mathrm{R}(0) \text { and } \mathrm{P}(2) \\
\text { spectra } \\
\end{array}$ \\
\hline \multicolumn{7}{|c|}{ Northern arm sources } \\
\hline IRS $1 \mathrm{~W}$ & Red & $35 \pm 20$ & Yes & No & Yes & Yes \\
\hline IRS 5 & Cool & $110 \pm 60$ & Yes & Yes & Yes & Yes \\
\hline \multirow[t]{2}{*}{ IRS 10W } & Cool & $80 \pm 60$ & Yes & Yes & Yes & Yes \\
\hline & Cool & $195 \pm 150$ & & & & \\
\hline IRS 21 & Hot & $-90 \pm 20$ & Yes & Yes & Yes & Yes \\
\hline \multicolumn{7}{|c|}{ The helium stars } \\
\hline IRS $16 \mathrm{C}$ & Hot & $125 \pm 30$ & Yes & No & No & Yes \\
\hline IRS 16SW & Hot & $453 \pm 4$ & Yes & No & No & Yes \\
\hline \multicolumn{7}{|c|}{ The bar and minicavity region } \\
\hline IRS $2 S$ & Cool & $107 \pm 20$ & Yes & Yes & Yes & Yes \\
\hline IRS 2L & Red & - & Yes & Yes & Yes & Yes \\
\hline IRS 3 & Red & - & Yes & Yes & Yes & Yes \\
\hline IRS $12 \mathrm{~N}$ & Cool & $-65 \pm 5$ & Yes & Yes & Yes & Yes \\
\hline IRS 13E & Hot & $45 \pm 60$ & Yes & Yes & Yes & Yes \\
\hline \multirow[t]{2}{*}{ IRS 29S } & Hot & $-93 \pm 20$ & Yes & No & Yes & Yes \\
\hline & Hot & $-164 \pm 2$ & & & & \\
\hline IRS 6W & Hot & $63 \pm 3$ & Yes & Yes & No & Yes \\
\hline
\end{tabular}

Notes. The spectral types and published radial velocities of the sources are given in Cols. 2 and 3, respectively. See Table 1 for references. For IRS $12 \mathrm{~N}$ we used the average of the values derived by Zhu et al. (2008) and Figer et al. (2003).

the approximately $-40 \mathrm{~km} \mathrm{~s}^{-1}$ line. However, these lines are respectively confirmed in the other available spectra of both IRS 16C and IRS 16SW. The helium stars are known not to be embedded in the minispiral. This may explain the absence of lines other than those absorbed in the four foreground clouds (at $-40,-60,-85$, and $-145 \mathrm{~km} \mathrm{~s}^{-1}$ ). This also confirms at the same time that the LOS lines are absorbed in a medium located outside the central parsec.

In general, most of the individual spectra show nonsaturated ${ }^{12} \mathrm{CO}$ lines at -40 and $-60 \mathrm{~km} \mathrm{~s}^{-1}$, unlike the IRS $1 \mathrm{~W}$ and IRS 3 spectra. This may indicate a variation of the ${ }^{12} \mathrm{CO}$ abundance in the corresponding foreground clouds across the field of view and towards the direction of the individual sources of the GC.

\section{Local absorptions in the Galactic center}

In the last column of Table 1, we list the velocities of additional lines that we detect in the spectra of the observed GC sources and that we assume to be associated with the individual sources. These lines are all detected at high transition levels (between $\mathrm{P}(3)$ and $\mathrm{P}(9)$ ) except for IRS $1 \mathrm{~W}$, where the detection is only obtained at low levels $(\mathrm{R}(0)$ and $\mathrm{P}(1))$ due to a lack of data at higher levels. As already mentioned, higher transition levels are excited at higher temperatures, implying that the gas at the origin of these absorptions is closer to the sources. In the following, for each of the sources we enumerate the observed local absorptions and discuss our findings. All results are summarized in Table 1.

IRS1W. We find absorptions at $35 \pm 15 \mathrm{~km} \mathrm{~s}^{-1}$ and $50 \pm 15 \mathrm{~km} \mathrm{~s}^{-1}$ in the spectra of the $\mathrm{R}(0)$ and $\mathrm{P}(1)$ transitions, respectively (see Fig. A.1). The first one is consistent with the value of the radial velocity of $35 \pm 20 \mathrm{~km} \mathrm{~s}^{-1}$ as derived by Paumard et al. (2006). We therefore assume that this absorption could be associated with the local medium of the source even though at this velocity we already concluded that the absorption is also attributed to the $50 \mathrm{~km} \mathrm{~s}^{-1}$ cloud.

IRS $2 L$ and IRS 13E. In the IRS 13E spectra shown in Fig. A.8, one can clearly distinguish two absorptions at about $\sim 50$ and $60 \pm 15 \mathrm{~km} \mathrm{~s}^{-1}$ in all available ro-vibrational transition spectra $(\mathrm{R}(0), \mathrm{P}(1), \mathrm{P}(2)$, and $\mathrm{P}(3))$. The $50 \mathrm{~km} \mathrm{~s}^{-1}$ line is due to the $50 \mathrm{~km} \mathrm{~s}^{-1}$ cloud as stated above and the $60 \mathrm{~km} \mathrm{~s}^{-1}$ absorption is consistent with the $45 \pm 60 \mathrm{~km} \mathrm{~s}^{-1}$ radial velocity of the source as measured by Genzel et al. (2000).

However, in all transition spectra of IRS 2L shown in Fig. A.10, we also find the two absorptions at around 50 and $60 \pm 15 \mathrm{~km} \mathrm{~s}^{-1}$. Therefore, the $60 \pm 15 \mathrm{~km} \mathrm{~s}^{-1}$ may also be coming from the local GC medium in the material of the IRS $2 \mathrm{~L}-$ IRS 13 complex. On the other hand, the $\sim 50 \pm 15 \mathrm{~km} \mathrm{~s}^{-1}$ is also probably attributed to the $50 \mathrm{~km} \mathrm{~s}^{-1} \mathrm{GC}$ cloud. Moreover, an additional absorption at $-105 \mathrm{~km} \mathrm{~s}^{-1}$ in the $\mathrm{R}(0), \mathrm{P}(1)$, and $\mathrm{P}(3)$ spectra of IRS $2 \mathrm{~L}$ is also detected. It may be a first determination of the proper radial velocity of the source that was not measured before or is possibly related to the minispiral material in the IRS $2 \mathrm{~L}-2 \mathrm{~S}-12 \mathrm{~N}$ region, since this velocity is also observed in the $\mathrm{R}(0)$ and $\mathrm{P}(1)$ line absorptions of IRS $2 \mathrm{~S}$ and IRS $12 \mathrm{~N}$.

IRS 2S. A faint absorption at $-110 \mathrm{~km} \mathrm{~s}^{-1}$ is visible in $\mathrm{R}(0), \mathrm{P}(1)$, and $\mathrm{P}(2)$ transition spectra but not in the $\mathrm{P}(3)$ spectrum (see Fig. A.11). It is not consistent with the $107 \pm 20 \mathrm{~km} \mathrm{~s}^{-1}$ radial velocity measured by Genzel et al. (2000). Hence, the 
absorption is possibly related to the gaseous stream of the minispiral ISM (see, e.g., the velocity field in Zhao et al. 2010) rather than to the emission line of the object IRS $2 \mathrm{~S}$ as it is also observed in the $\mathrm{R}(0)$ and $\mathrm{P}(1)$ spectra of IRS $12 \mathrm{~N}$ and the $\mathrm{R}(0)$, $\mathrm{P}(1)$, and $\mathrm{P}(2)$ spectra of IRS 2L.

IRS 3. The absorption at $60 \pm 15 \mathrm{~km} \mathrm{~s}^{-1}$ is present in the $\mathrm{R}(0), \mathrm{P}(1)$, and $\mathrm{P}(2)$ transition spectra and also in the ${ }^{12} \mathrm{CO} \mathrm{P}(6)$ and $\mathrm{P}(7)$ spectra (see Fig. A.2). There is no measurement of the radial velocity of this source in the literature. Our results therefore provide a first measurement of the radial velocity of the source even though an intervening cloud also absorbs at the same velocity (i.e., $\sim 50 \pm 15 \mathrm{~km} \mathrm{~s}^{-1}$ ) at low transition levels.

IRS 5. A prominent absorption is observed in all our spectra around $70 \pm 15 \mathrm{~km} \mathrm{~s}^{-1}$ (see Fig. A.3). This value is inside the error bars of the known radial velocity of this source of $110 \pm 60 \mathrm{~km} \mathrm{~s}^{-1}$ as given by Tanner et al. (2005).

IRS 6W. Two absorption lines at $-20 \pm \mathrm{km} \mathrm{s}^{-1}$ and $65 \pm \mathrm{km} \mathrm{s}^{-1}$ are present in the $\mathrm{P}(1), \mathrm{P}(2), \mathrm{P}(4)$, and $\mathrm{P}(5)$ spectra (see Fig. A.6). The $65 \mathrm{~km} \mathrm{~s}^{-1}$ absorption can be confused with the $50 \mathrm{~km} \mathrm{~s}^{-1}$ foreground absorption but such a velocity is consistent with the radial velocity of the source measured from emission lines (Zhu et al. 2008; Herbst et al. 1993). The absorption line at about $-20 \mathrm{~km} \mathrm{~s}^{-1}$ would then be associated with a gaseous component along the LOS, but it is unclear if it is located close to the GC.

IRS 10W. Towards this continuum source, the absorption features at $110 \pm 15$ and $210 \pm 15 \mathrm{~km} \mathrm{~s}^{-1}$ are not very prominent in the $\mathrm{P}(3)$ transition spectrum (see Fig. A.4) but are close to the emission line at $80 \pm 60 \mathrm{~km} \mathrm{~s}^{-1}$ in the minispiral gaseous flow (Zhao et al. 2010) and the $195 \pm 150 \mathrm{~km} \mathrm{~s}^{-1}$ line possibly associated with the star (Tanner et al. 2005). In addition, our spectra show a faint absorption at $-105 \pm 15 \mathrm{~km} \mathrm{~s}^{-1}$ that has to be confirmed at higher $\mathrm{S} / \mathrm{N}$.

IRS 12N. This source is the best example we have, showing that we detect ${ }^{12} \mathrm{CO}$ gas at the velocity of the source and consequently that it is directly associated with the source. Absorption features at $-85 \pm 15 \mathrm{~km} \mathrm{~s}^{-1}$ are detected in most of the observed transition lines $(\mathrm{R}(0), \mathrm{P}(1), \mathrm{P}(3), \mathrm{P}(4), \mathrm{P}(6), \mathrm{P}(7)$ and $\mathrm{P}(9)$ ) (see Fig. A.9). This velocity is consistent with the radial velocity of the source of about $-65 \pm 2 \mathrm{~km} \mathrm{~s}^{-1}$ combining the values given by Zhu et al. (2008) and Figer et al. (2003). Even though this velocity is also rather coincident with the $-85 \mathrm{~km} \mathrm{~s}^{-1}$ LOS presumably cold cloud we already mentioned, it is also probably associated with warmer material probably closer to the source since high transition levels are detected as well. In addition, as mentioned previously in the IRS $2 \mathrm{~S}$ and $2 \mathrm{~L}$ paragraphs, an absorption observed at $-110 \pm 15 \mathrm{~km} \mathrm{~s}^{-1}$ in the $\mathrm{R}(0)$ and $\mathrm{P}(1)$ spectra is probably associated with the material of the minispiral in the IRS $2 \mathrm{~L}-2 \mathrm{~S}-12 \mathrm{~N}$ region.

IRS $16 C$ and SW. The IRS 16 sources (C and SW) are in the northern part of the IRS 16 association of bright He stars. IRS $16 \mathrm{C}$ is offset by about 2-3 arcseconds from the bar feature in the minispiral and IRS $16 \mathrm{SW}$ just north of or slightly within the bar (see Fig. 2). As mentioned in Sect. 3.5, we find absorption features in the ${ }^{12} \mathrm{CO} \mathrm{R}(0)$ transition that we already attributed to LOS clouds. All other higher ro-vibrational transitions we probed do not show any strong absorption line that may be characteristic of local absorption (see Figs. A.12 and A.13).
IRS 21. An absorption feature at $-60 \pm 15 \mathrm{~km} \mathrm{~s}^{-1}$ is detected in the $\mathrm{P}(3)$ transition spectrum (see Fig. A.5) that can be confused with the foreground cloud discussed in Sect. 3.1. Within the uncertainties it is also consistent with the radial velocity of the source of $-90 \pm 20 \mathrm{~km} \mathrm{~s}^{-1}$ as obtained by Genzel et al. (2000). Also within the uncertainties, an absorption at $55 \pm$ $15 \mathrm{~km} \mathrm{~s}^{-1}$ visible in the $\mathrm{R}(0), \mathrm{P}(1), \mathrm{P}(2)$, and $\mathrm{P}(3)$ ro-vibrational spectra may be consistent with the $50 \mathrm{~km} \mathrm{~s}^{-1}$ cloud.

IRS 29. The spectra of IRS 29 in the $\mathrm{R}(0)$ and $\mathrm{P}(1)$ transitions show the LOS absorption at $-60 \mathrm{~km} \mathrm{~s}^{-1}$ but this absorption is also visible in the $\mathrm{P}(3)$ higher-transition spectrum. Since it is compatible with the value of the radial velocity found by Genzel et al. (2000), we assume that this absorption is also associated with the source.

\section{Conclusion and discussion}

In this paper we present the results derived from our highspectral-resolution CRIRES observations of 13 IR sources located in the central parsec of the Galaxy. The data were obtained around the ${ }^{12} \mathrm{CO} \mathrm{R}(0), \mathrm{P}(1), \mathrm{P}(2), \mathrm{P}(3), \mathrm{P}(4), \mathrm{P}(5)$, $\mathrm{P}(6), \mathrm{P}(7)$, and $\mathrm{P}(9)$ transition lines and provide the first evidence of a complex structure in the ISM along the LOS and in the close environment of the central sources. We have found evidence for four foreground cold clouds at radial velocities $\mathrm{V}_{\mathrm{LSR}}$ of the order of $-145,-85,-60$, and $-40 \pm 15 \mathrm{~km} \mathrm{~s}^{-1}$ that show absorptions in the lower transition levels from $\mathrm{R}(0)$ to $\mathrm{P}(2)$ and in all the observed spectra. We also found evidence for two other cold clouds at radial velocities $\mathrm{V}_{\mathrm{LSR}}$ of the order of -185 and $-130 \pm 15 \mathrm{~km} \mathrm{~s}^{-1}$ that seem to be closer to the GC since the absorption lines at the velocities are not present in all the spectra. Specifically, these lines are absent in the spectra of the helium IRS 16 sources that are away from the minispiral structure, indicating that the corresponding cloud may be associated with the minispiral material with a temperature around $300 \mathrm{~K}$ (e.g., Garcia-Marin et al. 2011; Kunneriath et al. 2012; Serabyn \& Guesten 1986).

The same argument leads to the conclusion that a closer cloud at a velocity of $-210 \pm 15 \mathrm{~km} \mathrm{~s}^{-1}$, and maybe another one also at $60 \pm 15 \mathrm{~km} \mathrm{~s}^{-1}$, is probably present in front of the IRS 13, IRS 2L complex as the spectra of these sources show absorption lines at these velocities. Moreover, in all sources we find an absorption in velocity range of $50-60 \mathrm{~km} \mathrm{~s}^{-1}$, possibly associated with the $50 \mathrm{~km} \mathrm{~s}^{-1}$ cloud in the GC region. This opens up the possibility that the $50 \mathrm{~km} \mathrm{~s}^{-1}$ cloud has a low-density extension in front of the GC giving rise to the observed absorptions (see detailed discussion on the $50 \mathrm{~km} \mathrm{~s}^{-1}$ cloud extension further in the conclusion). This provides us with a clearer picture of the distribution of the different intervening clouds. In Fig. 5, we propose a rough projection of the distribution of these clouds in the field of view.

We also detected additional absorption lines in the spectra of most of the individual sources at high transition levels (from $\mathrm{P}(3)$ to $\mathrm{P}(9)$ ). For most of them, the measured velocities fit the radial velocities of the sources themselves as obtained from the literature. This indicates that the absorptions are taking place in the very close environment of the sources at the temperatures deduced from these transition lines of a few tens of Kelvin up to 300 K (e.g., Moneti et al. 2001; Garcia-Marin et al. 2011; Kunneriath et al. 2012; Serabyn \& Guesten 1986). At the same time, we were able to determine proper radial velocities of IRS $2 \mathrm{~L}$ and IRS $13 \mathrm{~N}$ that have not been measured previously. 


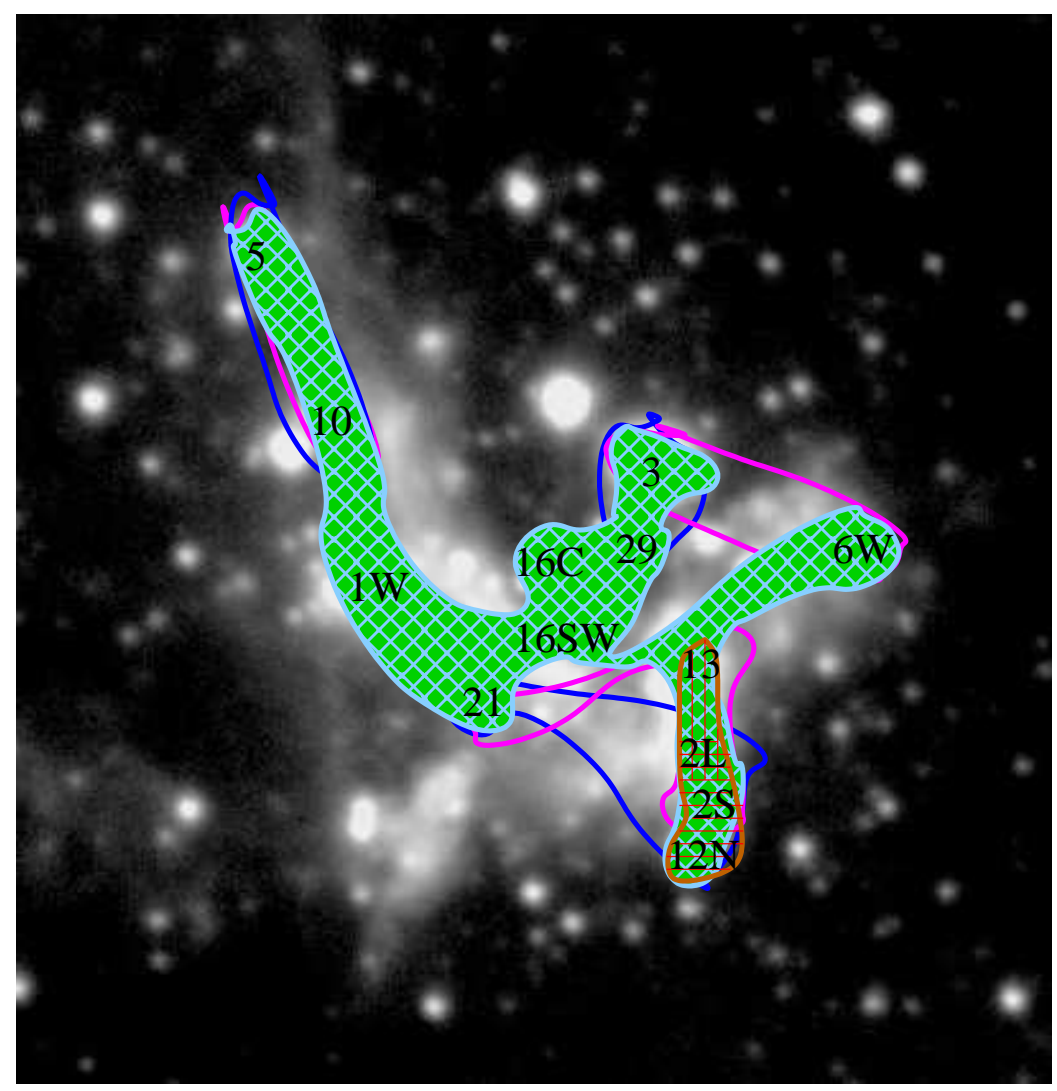

Fig. 5. Schematic view of the bright-continuum-emitting regions in the central parsec that are affected by absorption of $-145,-85,-60,-40$ and $50 \mathrm{~km} \mathrm{~s}^{-1}$ clouds (in light blue dashed with green), the $-185 \mathrm{~km} \mathrm{~s}^{-1}$ cloud (in magenta), the $-130 \mathrm{~km} \mathrm{~s}^{-1}$ cloud (in blue), the $-210 \mathrm{~km} \mathrm{~s}^{-1}$ IRS 13-2L-2S-12N complex cloud (in brown), and the 60 and $-110 \mathrm{~km} \mathrm{~s}^{-1}$ minispiral material (dashed red vertically and horizontally, respectively).
The fact that for a number of sources the low $\mathrm{R}$ and $\mathrm{P}$ rovibrational absorption lines are also at the radial velocities of the sources implies that the absorptions either take place in colder LOS clouds (which is less likely) or it may provide further evidence for the fact that low-temperature material can in fact survive in clouds during the short (in comparison to the evaporation timescale) transient time through the central cluster as we claimed in our previous works (e.g., Moultaka et al. 2015a,b).

The proposed extension of the $50 \mathrm{~km} \mathrm{~s}^{-1}$ cloud. As mentioned above, since all of the continuum sources in the central parsec show an absorption at $50 \mathrm{~km} \mathrm{~s}^{-1}$, this may point toward the existence of an extension of the $50 \mathrm{~km} \mathrm{~s}^{-1}$ cloud which must be floating by the GC northeast of the center (see Figs. 1 and 6 presenting a possible 3D distribution of this extension along the LOS towards the GC). Alternatively, the absorption towards the central continuum sources shows up incidentally at approximately the same velocity as the $50 \mathrm{~km} \mathrm{~s}^{-1}$ cloud.

The presence of an $\mathrm{H}_{2} \mathrm{CO}$ absorption in front of Sgr A East but not Sgr A West lead to the conclusion that the $50 \mathrm{~km} \mathrm{~s}^{-1}$ cloud is located behind Sgr A West (Whiteoak et al. 1974). This finding has been supported by several other investigations as was pointed out by Geballe et al. (1989) and Goto et al. (2014). The abundance ratio between $\mathrm{CO}$ and $\mathrm{H}_{2} \mathrm{CO}$ is of the order of $10^{54}$. The ratio of the absorption line strengths between Sgr A East and West is only of the order of $12^{5}$. Hence, in a case of low to moderate optical depth, the presence of $\mathrm{CO}$ absorption in the absence of $\mathrm{a} \mathrm{H}_{2} \mathrm{CO}$ absorption in front of $\mathrm{Sgr} \mathrm{A}$ West is relatively conceivable.

\footnotetext{
4 With respect to molecular hydrogen, Pauls et al. (1996) give a Formaldehyde abundance towards Sgr A of a few times $10^{-10}$.

5 Whiteoak et al. (1974) find $\mathrm{H}_{2} \mathrm{CO}$ optical depths toward Sgr A East 2.5 and less than 0.03 toward Sgr A West in a $20^{\prime \prime} \times 40^{\prime \prime}$ beam.
}

However, Geballe et al. (1989) already detected CO absorption features in the spectra of IRS 3 and IRS 7 speculating that these absorptions may be due to the $50 \mathrm{~km} \mathrm{~s}^{-1}$ cloud. At that point the conclusion was unequivocal as the velocity of the western edge of the CND is also close to $50 \mathrm{~km} \mathrm{~s}^{-1}$. Also, Goto et al. (2014) suggested that the $60 \mathrm{~km} \mathrm{~s}^{-1}$ absorption observed in their spectra is probably associated with the CND. Moreover, Geballe et al. (1989) did not detect $50 \mathrm{~km} \mathrm{~s}^{-1}$ absorption towards IRS 1 and IRS 2. Our detection of absorption close to $50 \mathrm{~km} \mathrm{~s}^{-1}$ towards most continuum sources implies that a $50 \mathrm{~km} \mathrm{~s}^{-1}$ cloud filament indeed lies in front of the central stellar cluster. If the absorptions were predominantly from the CND then in the eastern region of the stellar cluster we would expect them to occur rather at -20 to $-50 \mathrm{~km} \mathrm{~s}^{-1}$ (e.g., Amo-Baladron et al. 2011). Hence, we are forced to conclude that a $50 \mathrm{~km} \mathrm{~s}^{-1}$ cloud is in front of the entire central stellar cluster and may be associated with the $50 \mathrm{~km} \mathrm{~s}^{-1}$ cloud whose bulk however is located behind Sgr A West.

One may question how a dynamically stabile configuration can be explained that allows for an extension of the $50 \mathrm{~km} \mathrm{~s}^{-1}$ cloud into the LOS towards the continuum sources in the GC close to Sgr A. To answer this question, we can compare the radial motion of $50 \mathrm{~km} \mathrm{~s}^{-1}$ with the expected orbital velocity at the distance of about $5 \mathrm{pc}$ to the GC implied by Lee et al. (2008). Using the enclosed mass plot given by Schödel et al. (2003), we find an enclosed mass of $M_{6}=M /\left(10^{6} M_{\odot}\right) \sim 10$. With the relation $v\left(\mathrm{~km} \mathrm{~s}^{-1}\right)=2.07 \sqrt{M_{6} / r(\mathrm{kpc})}$, this results in an expected orbital velocity of about $92 \mathrm{~km} \mathrm{~s}^{-1}$. This implies that the proper motion of the $50 \mathrm{~km} \mathrm{~s}^{-1}$ cloud is at least $77 \mathrm{~km} \mathrm{~s}^{-1}$ or higher. Hence, the entire $50 \mathrm{~km} \mathrm{~s}^{-1}$ cloud including its extension suggested by our absorption line profiles may be moving, for example, towards the NW or SE, passing by the GC. This situation is shown in the top two panels of Fig. 6. 

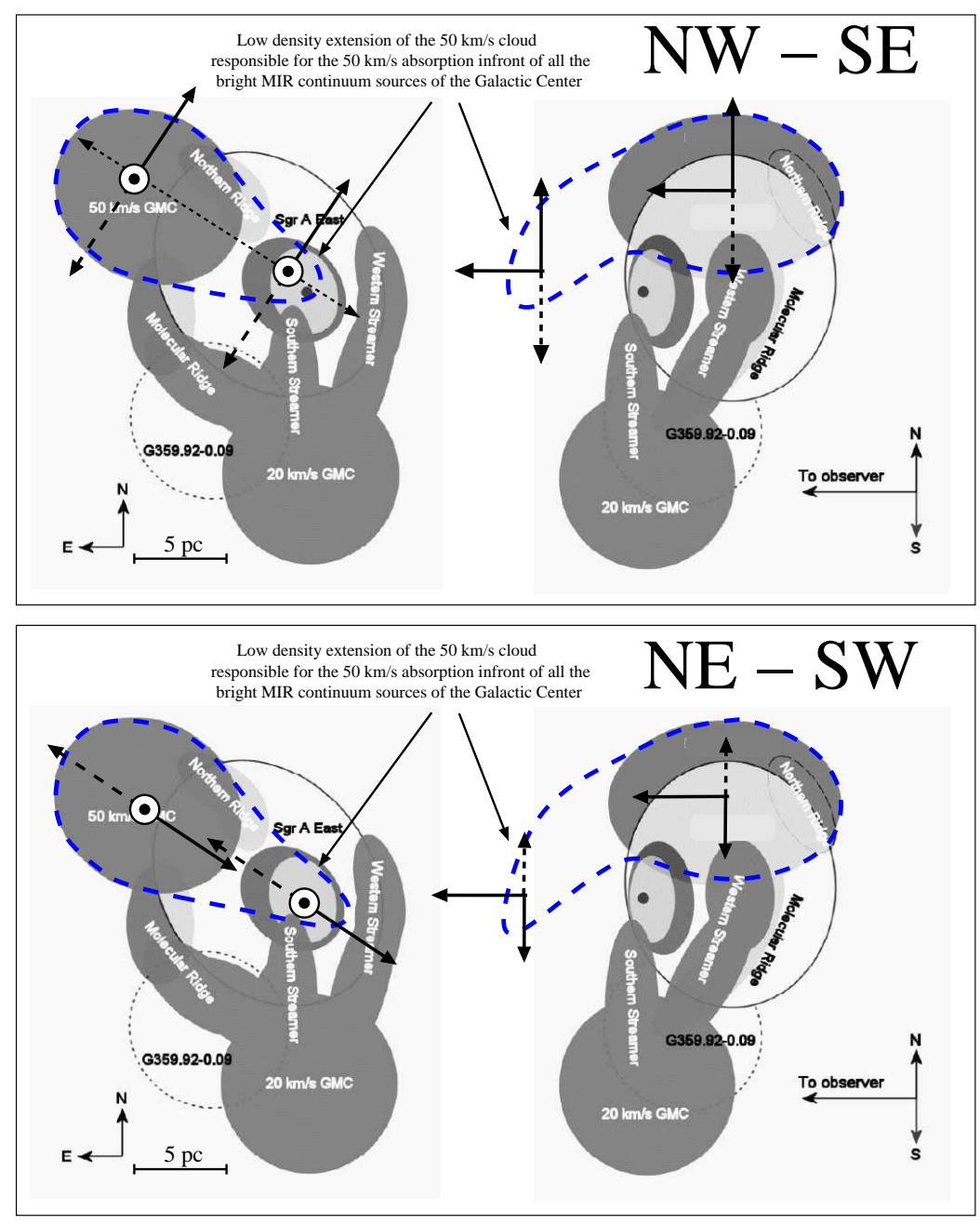

Fig. 6. Schematic view of the $50 \mathrm{~km} \mathrm{~s}^{-1}$ cloud. It is one of the closest cloud complexes to the GC. Following Lee et al. (2008) we show the relative locations of some of the clouds that are quite close to the LOS. The circumference of the $50 \mathrm{~km} \mathrm{~s}^{-1}$ cloud and its possible extension have been indicated in light blue. In the right panels, the radial velocity vector is shown as a narrow peak pointing towards the observer. In the left panels, the radial velocity vector is shown as a solid horizontal vector pointing towards the observer. The approximate linear scale is indicated. Top panel: situation if the $50 \mathrm{~km} \mathrm{~s}^{-1}$ cloud and its extension have proper motions towards the NW (bold solid line) or SE (bold dashed line). We have drawn the radial and proper motion vectors (and their projections) in both top panel views. The direction perpendicular to the orbital plane of the bypassing $50 \mathrm{~km} \mathrm{~s}^{-1}$ cloud complex is shown as a short dashed line. Bottom panel: situation for a proper motion in NE (bold solid line) or SW (bold dashed line).
As shown in the bottom two panels of Fig. 6, a proper motion in the NE or SW direction is less likely. If the $50 \mathrm{~km} \mathrm{~s}^{-1}$ cloud was on a NE trajectory (dashed line in the bottom panel of Fig. 6) it would come almost straight from the central cluster region. A survival of that passage or the presence of the nevertheless rather fragile and transient CND (Requena-Torres 2012) would be very unlikely. Flying towards the SW would imply that the $50 \mathrm{~km} \mathrm{~s}^{-1}$ cloud is heading towards the central cluster region (bold line in the bottom panel of Fig. 6). In both cases one would expect a significant velocity gradient and a velocity difference between the main cloud and its extension - also in the radial direction but this is not the case.

However, the flight directions NW and SE could be realized as a periapse passage of the $50 \mathrm{~km} \mathrm{~s}^{-1}$ cloud. In this case, the main cloud and its extension could be passing by the central region in one common orbital plane at approximately the same distance and argument of periapsis (top panels in Fig 6). The radial velocities would be very similar and differences between the $50 \mathrm{~km} \mathrm{~s}^{-1}$ cloud and its extension would mainly be dominated by a relatively small velocity gradient perpendicular to the orbital plane of this cloud system. In this scenario, part of the cloud could be in front of the central cluster region with the same radial velocity as the bulk of the cloud.

A very good candidate for this $50 \mathrm{~km} \mathrm{~s}^{-1}$ cloud section in front of the central stellar cluster is the central association (CA) found in $50 \mathrm{~km} \mathrm{~s}^{-1}$ molecular line emission with ALMA (Moser et al. 2017). This cloud complex is most likely identical to the
$50 \mathrm{~km} \mathrm{~s}^{-1} \mathrm{OH}$ absorption component found by Karlsson et al. (2015). In both cases, the gas covers the central region with an extent of about 20 arcseconds including Sgr A* and the central stellar cluster. The arrangement of $\mathrm{OH}$-absorbing gas at $59 \mathrm{~km} \mathrm{~s}^{-1}$ shown by Karlsson et al. (2015) in their Fig. 1 (see also Fig. 12 by Moser et al. 2017) is very similar to our proposed extent of the $50 \mathrm{~km} \mathrm{~s}^{-1}$ cloud (blue dashed contour in the top left panel of Fig. 6). This is particularly true if one considers the fact that the component in front of the central stellar cluster and the $50 \mathrm{~km} \mathrm{~s}^{-1}$ cloud region are linked at velocities between 20 and $50 \mathrm{~km} \mathrm{~s}^{-1}$ as shown in the $\mathrm{OH}$ absorption channel maps by Karlsson et al. (2015) in their Fig. 2.

In this paper, we have shown that the continuum sources within the central parsec are ideally suited to studying the physical conditions and the location of the absorbing clouds. For the mostly extended dusty sources, future observations with higher angular resolution and point-source sensitivity will potentially allow us to spatially discriminate between absorption against the compact stellar component and the more extended part of the structure that might partially come from the mini-spiral gas flow. Higher sensitivity will also allow for the absorption towards fainter sources to be mapped out such that the spatial distribution of the absorbing gas clouds can be determined in a more complete way. Right now we can only comment on the absorption towards relatively bright sources. The absorbing clouds may however have a larger extent and the $50 \mathrm{~km} \mathrm{~s}^{-1}$ absorption may actually cover the entire field (see Fig. 6). Suitable instruments 
for future point-source-sensitive observations are MATISSE at the VLT (Lopez et al. 2018) and METIS at the EELT (Brandl et al. 2018). Instruments that permit further studies of the absorption towards more extended source components are VISIR at the VLT (Lagage et al. 2003) and MIRI aboard the JWST (Garcia Marin et al. 2018; Wright et al. 2010).

\section{References}

Amo-Baladron, M. A., Martin-Pintado, J., \& Martin, S. 2011, A\&A, 526, A54 Brandl, B. R., Absil, O., Agócs, T., et al. 2018, SPIE, 10702, 107021

Chiar, J. E., Tielens, A. G. G. M., Whittet, D. C. B., et al. 2000, ApJ, 537, 749

Chiar, J. E., Adamson, A. J., Pendleton, Y. J., et al. 2002, ApJ, 570, 198

Chiar, J. E., Adamson, A. J., Whittet, D. C. B., et. al. 2006, ApJ, 651, 268

Eckart, A., Moultaka, J., Viehmann, T., Straubmeier, C., \& Mouawad, N. 2004, ApJ, 602, 760

Figer, D. F., Gilmore, D., Kim, S. S., et al. 2003, ApJ, 599, 1139

Garcia-Marin, M., Eckart, A., Weiss, A., et al. 2011, ApJ, 738, 158

Garcia Marin, M., Rieke, G., Ressler, M., et al. 2018, SPIE, 10704, 107041

Ghez, A. M., Duchene, G., Matthews, K., et al. 2003, ApJ, 586, L127

Geballe, T. R. 1986, A\&A, 162, 248

Geballe, T. R., Baas, F., \& Wade, R. 1989, A\&A, 208, 255

Genzel, R., Pichon, C., Eckart, A., Gerhard, O. E., \& Ott, T. 2000, MNRAS, 317, 348

Goto, M., Geballe, T. R., Indriolo, N., et al. 2014, ApJ, 786, 96

Guesten, R., Genzel, R., Wright, M. C. H., et al. 1987, ApJ, 318, 124

Herbst, T. M., Beckwith, S. V. W., Forrest, W. J., \& Pipher, J. L. 1993, AJ, 105, 956

Kaifu, N., Kato, T., \& Iguchi, T. 1972, Nature, 238, 105

Karlsson, R., Sandqvist, Aa., Fathi, K., \& Martín, S. 2015, A\&A, 582, A118

Kausch, W., Noll, S., Smette, A., et al. 2015, A\&A, 576, A78

Kunneriath, D., Eckart, A., Vogel, S. N., et al. 2012, A\&A, 538, A127

Lacy, J. H., Baas, F., Allamandola, L. J., et al. 1984, ApJ, 276, 533

Lagage, P.-O., Pel, J.-W., Claret, A., et al. 2003, SPIE, 4841, 923

Lee, S., Pak, S., Choi, M., Davis, C. J., \& Geballe, T. R. 2008, ApJ, 674, 247

Lopez, B., Lagarde, S., Matter, A., et al. 2018, SPIE, 10701, 107010

Lu, X., Zhang, Q., Kauffmann, J., et al. 2017, ApJ, 839, 1

Martins, F., Trippe, S., Paumard, T., et al. 2006, ApJ, 649, L103

McFadzean, A. D., Whittet, D. C. B., Longmore, A. J., Bode, M. F., \& Adamson, A. J. 1989, MNRAS, 241, 873

Moneti, A., Cernicharo, J., \& Pardo, J. R. 2001, ApJ, 549, L203
Moser, L., Sanchez-Monge, A., Eckart, A., et al. 2017, A\&A, 603, A68

Mossoux, E., \& Eckart, A. 2018, MNRAS, 474, 3787

Moultaka, J., Eckart, A., Viehmann, T., et al. 2004, A\&A, 425, 529

Moultaka, J., Eckart, A., Schödel, R., Viehmann, T., \& Najarro, F. 2005, A\&A, 443, 163

Moultaka, J., Eckart, A., \& Schödel, R. 2009, ApJ, 703, 1635

Moultaka, J., Eckart, A., \& Sabha, N. 2015a, MNRAS, 448, 3363

Moultaka, J., Eckart, A., \& Muzic, K. 2015b, ApJ, 806, 202

Muzic, K., Eckart, A., Schödel, R., Meyer, L., \& Zensus, A. 2007, A\&A, 469, 993

Oka, T., Geballe, T. R., Goto, M., Usuda, T., \& McCall, B. J. 2005, ApJ, 632, 882

Ott, T., Eckart, A., \& Genzel, R. 1999, ApJ, 523, 248

Pauls, T., Johnston, K. J., \& Wilson, T. L. 1996, ApJ, 461, 223

Paumard, T., Maillard, J. P., Morris, M., \& Rigaut, F. 2001, A\&A, 366, 466

Paumard, T., Maillard, J.-P., \& Morris, M. 2004, A\&A, 426, 81

Paumard, T., Genzel, R., Martins, F., et al. 2006, ApJ, 643, 1011

Perger, M., Moultaka, J., Eckart, A., et al. 2008, A\&A, 478, 127

Requena-Torres, M. A., Güsten, R., Weiß, A., et al. 2012, A\&A, 542, L21

Schödel, R., Ott, T., Genzel, R., et al. 2003, ApJ, 596, 1015

Schödel, R., Eckart, A., Alexander, T., et al. 2007, A\&A, 469, 125

Scoville, N. Z. 1972, ApJ, 175, L127

Scoville, N. Z., Stolovy, S. R., Rieke, M., Christopher, M., \& Yusef-Zadeh, F. 2003, ApJ, 594, 294

Serabyn, E., \& Guesten, R. 1986, A\&A, 161, 334

Smette, A., Sana, H., Noll, S., et al. 2015, A\&A, 576, A77

Sofue, Y. 1995, PASJ, 47, 551

Sutton, E. C., Danchi, W. C., Jaminet, P. A., \& Masson, C. R. 1990, ApJ, 348, 503

Tanner, A. M., Ghez, A. M., Morris, M., \& Becklin, E. E. 2003, Astron. Nachr., 324,597

Tanner, A., Ghez, A. M., Morris, M. R., \& Christou, J. C. 2005, ApJ, 624, 742

Takekawa, S., Oka, T., \& Tanaka, K. 2017, ApJ, 834, 121

Tegler, S. C., Weintraub, D. A., Allamandola, L. J., et al. 1993, ApJ, 411, 260

Tsuboi, M., Kitamura, Y., Uehara, K., et al. 2018, PASJ, 70, 85

Uehara, K., Tsuboi, M., Kitamura, Y., et al. 2017, IAU Symp. 322, 162

Whiteoak, J. B., Rogstad, D. H., \& Lockhart, I. A. 1974, A\&A, 36, 245

Whittet, D. C. B., Boogert, A. C. A., Gerakines, P. A., et al. 1997, ApJ, 490, 729

Wright, M. C. H., Coil, A. L., McGary, R. S., et al. 2001, ApJ, 551, 254

Wright, G. S., Rieke, G., Böker, T., et al. 2010, SPIE, 7731, 77310

Zhao, J.-H., Blundell, R., Moran, J. M., et al. 2010, ApJ, 723, 1097

Zhu, Q., Kudritzki, R. P., Figer, D. F., Najarro, F., \& Merritt, D. 2008, ApJ, 681, 1254 


\section{Appendix A: Spectra of the Galactic center IR sources}
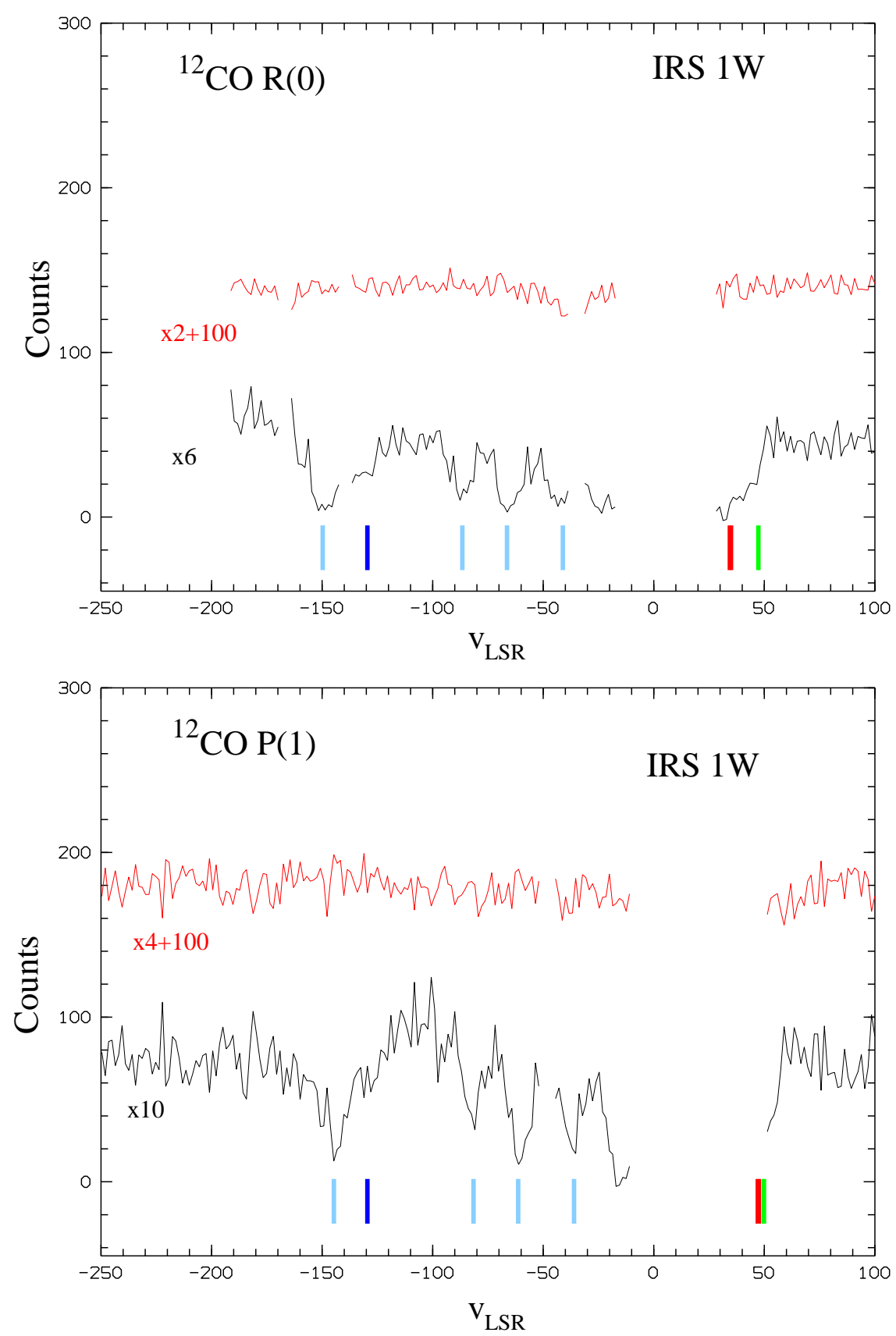

Fig. A.1. Spectra (in black) of IRS $1 \mathrm{~W}$ in the ${ }^{12} \mathrm{CO} \mathrm{R}(0)$ and $\mathrm{P}(1)$ line transitions. Also shown (in red) are the spectra of the standard stars observed close to the science observation to correct for telluric lines. The blanked areas correspond to regions in the spectrum where the telluric corrections were not successful. The red arrows indicate the local absorptions at velocities listed in Table 1 . The other colored arrows indicate the absorptions at the same velocities as in Figs. 3 and 4. The scaling and/or shifting factors of the spectra are given in red and black for the standard star and the GC object, respectively. 

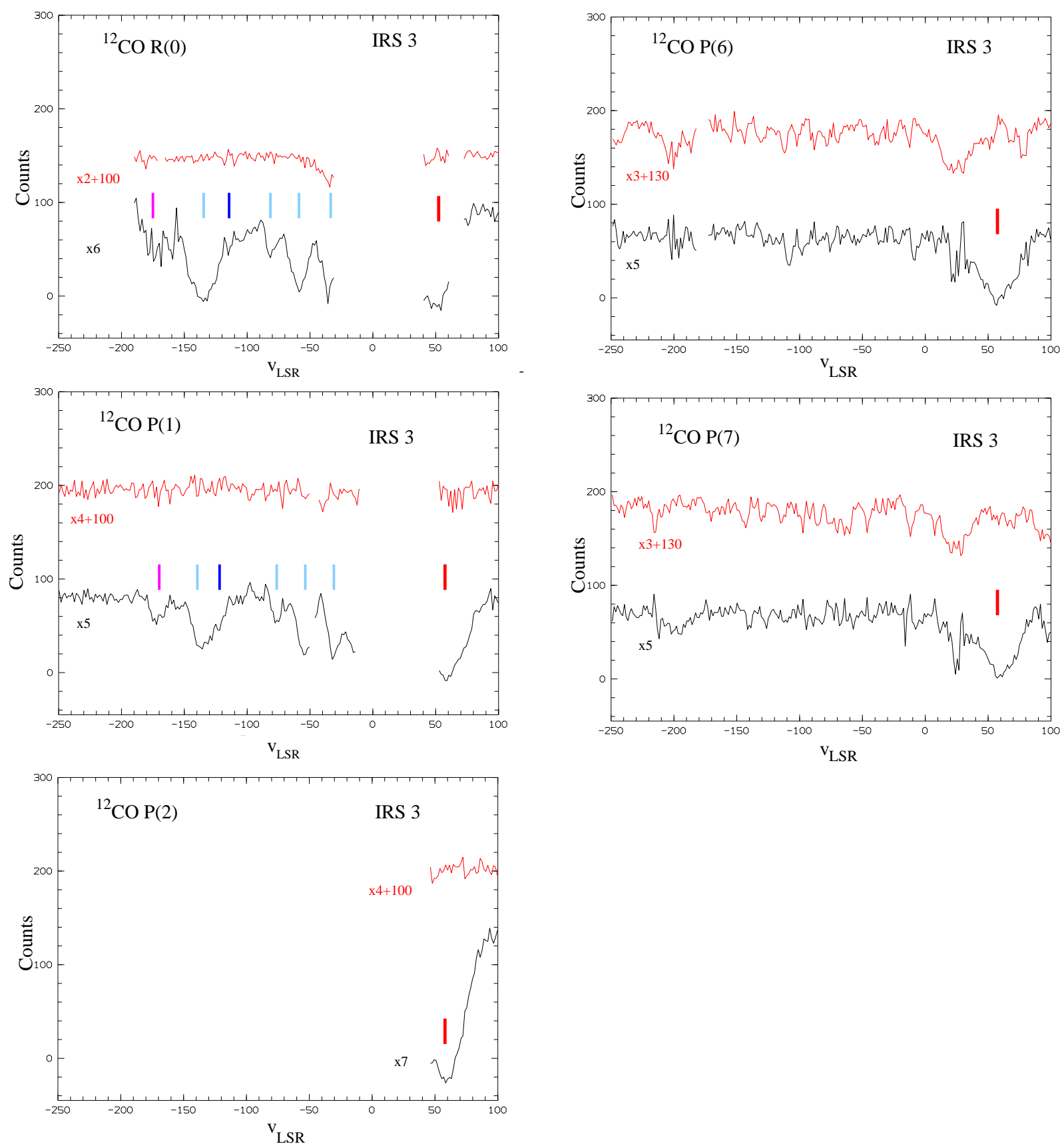

Fig. A.2. Spectra (in black) of IRS 3 in the ${ }^{12} \mathrm{CO} R(0), P(1), P(2), P(6)$ and $\mathrm{P}(7)$ transitions. Also shown (in red) are the spectra of the standard stars observed close to the science observation to correct for telluric lines. The blanked areas correspond to regions in the spectrum where the telluric corrections were not successful. The red arrows indicate the local absorptions at velocities listed in Table 1 . The other colored arrows indicate the absorptions at the same velocities as in Figs. 3 and 4. The scaling and/or shifting factors of the spectra are given in red and black for the standard star and the GC object, respectively. 
J. Moultaka et al.: CRIRES observations of the GC
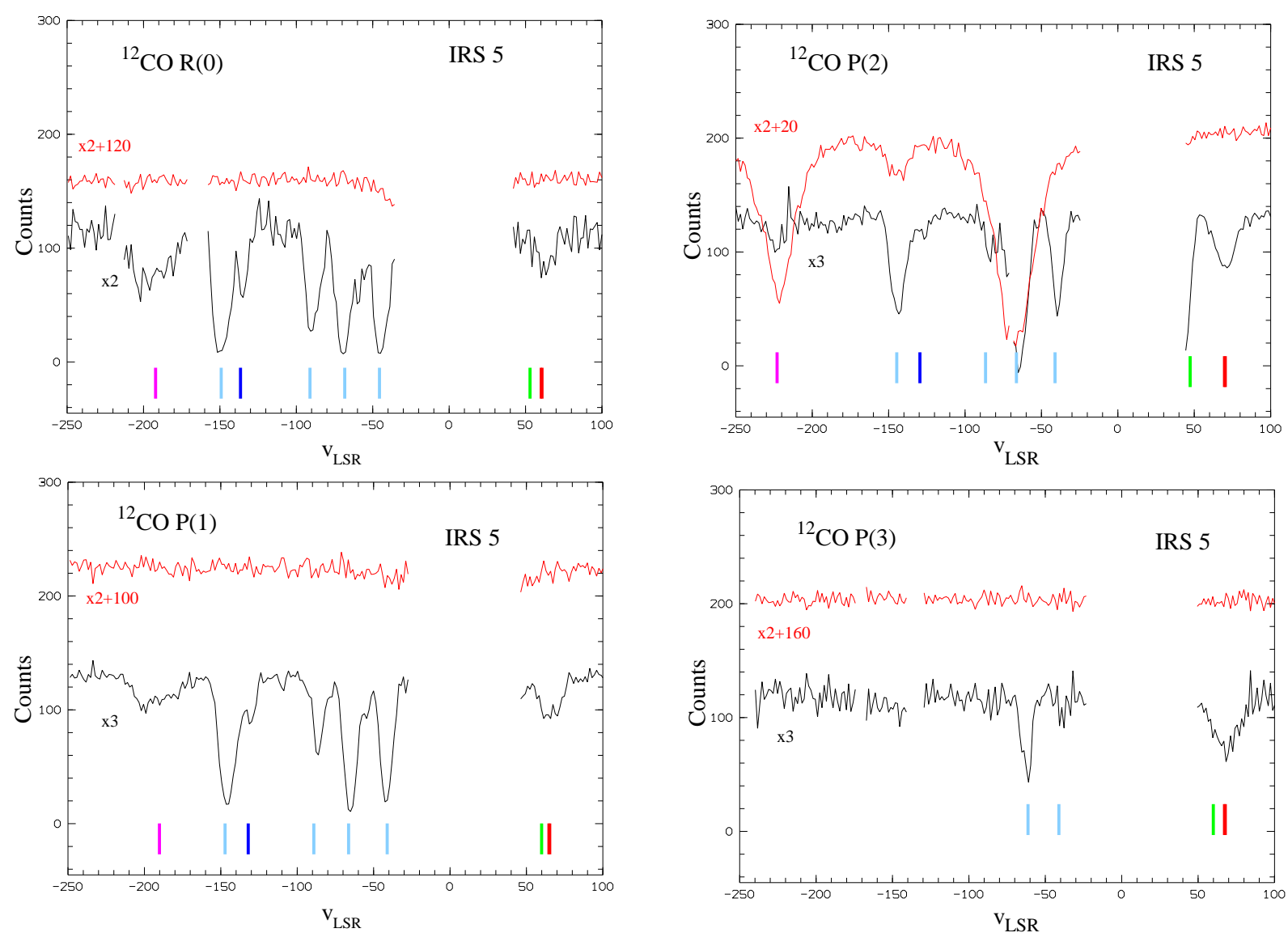

Fig. A.3. Spectra (in black) of IRS 5 in the ${ }^{12} \mathrm{CO} \mathrm{R}(0), \mathrm{P}(1), \mathrm{P}(2)$ and $\mathrm{P}(3)$ transitions. Also shown (in red) are the spectra of the standard stars observed close to the science observation to correct for telluric lines. The blanked areas correspond to regions in the spectrum where the telluric corrections were not successful. The red arrows indicate the local absorptions at velocities listed in Table 1 . The other colored arrows indicate the absorptions at the same velocities as in Figs. 3 and 4. The scaling and/or shifting factors of the spectra are given in red and black for the standard star and the GC object, respectively. 

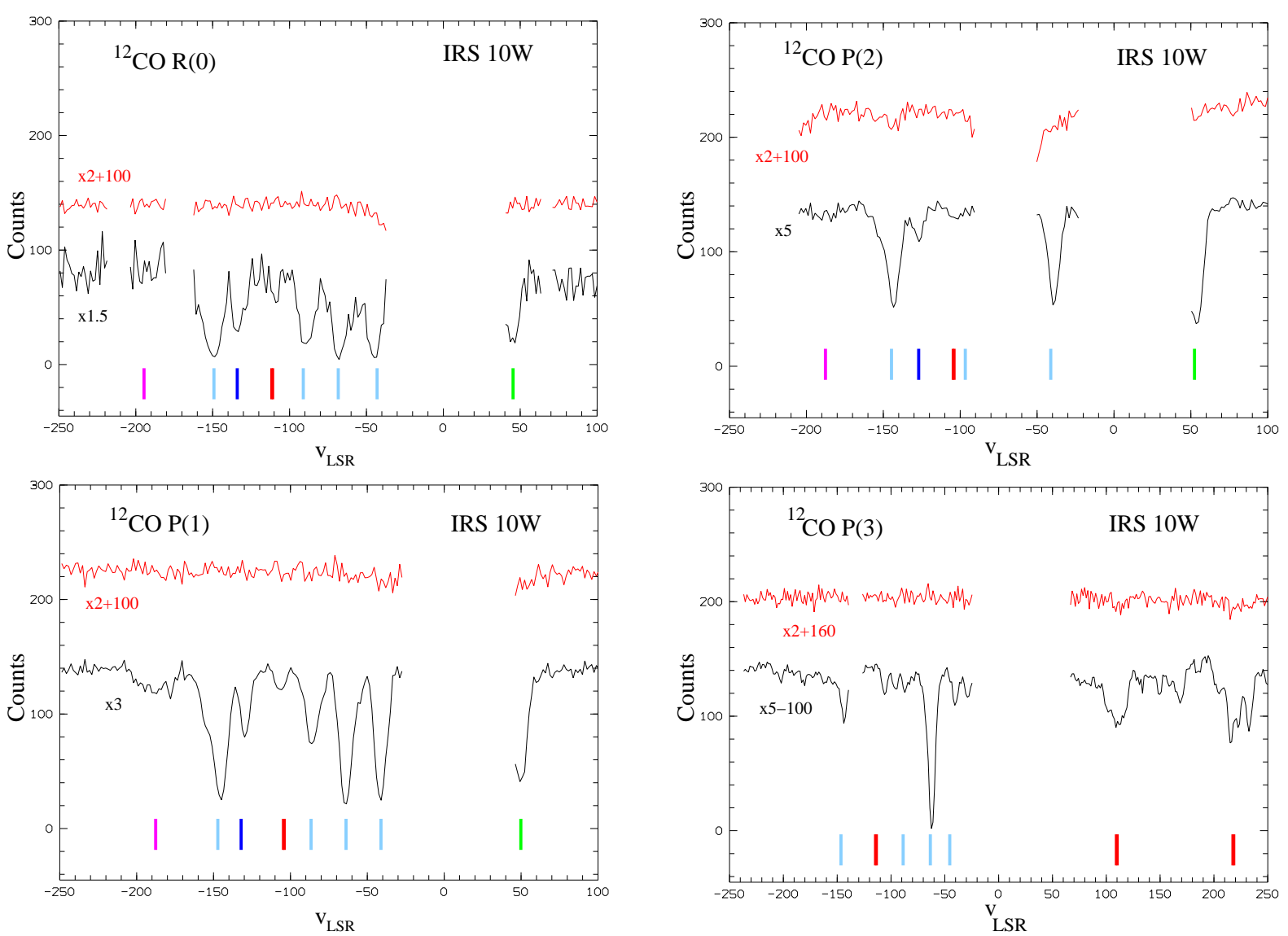

Fig. A.4. Spectra (in black) of IRS $10 \mathrm{~W}$ in the ${ }^{12} \mathrm{CO} R(0), \mathrm{P}(1), \mathrm{P}(2)$ and $\mathrm{P}(3)$ transitions. Also shown (in red) are the spectra of the standard stars observed close to the science observation to correct for telluric lines. The blanked areas correspond to regions in the spectrum where the telluric corrections were not successful. The red arrows indicate the local absorptions at velocities listed in Table 1 . The other colored arrows indicate the absorptions at the same velocities as in Figs. 3 and 4. The scaling and/or shifting factors of the spectra are given in red and black for the standard star and the GC object, respectively. 
J. Moultaka et al.: CRIRES observations of the GC
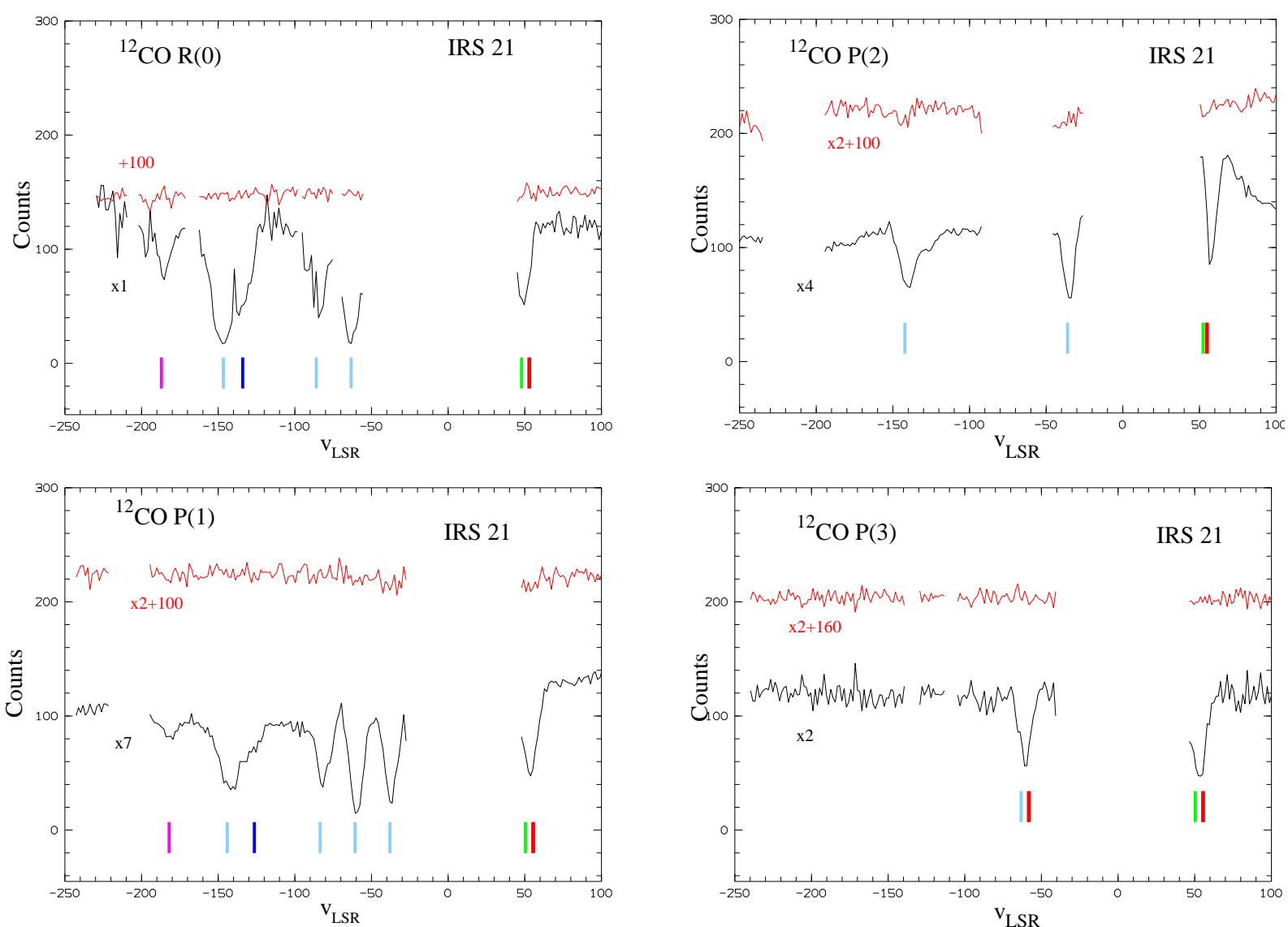

Fig. A.5. Spectra (in black) of IRS 21 in the ${ }^{12} \mathrm{CO} R(0), \mathrm{P}(1), \mathrm{P}(2)$ and $\mathrm{P}(3)$ transitions. Also shown (in red) are the spectra of the standard stars observed close to the science observation to correct for telluric lines. The blanked areas correspond to regions in the spectrum where the telluric corrections were not successful. The red arrows indicate the local absorptions at velocities listed in Table 1 . The other colored arrows indicate the absorptions at the same velocities as in Figs. 3 and 4. The scaling and/or shifting factors of the spectra are given in red and black for the standard star and the GC object, respectively. 

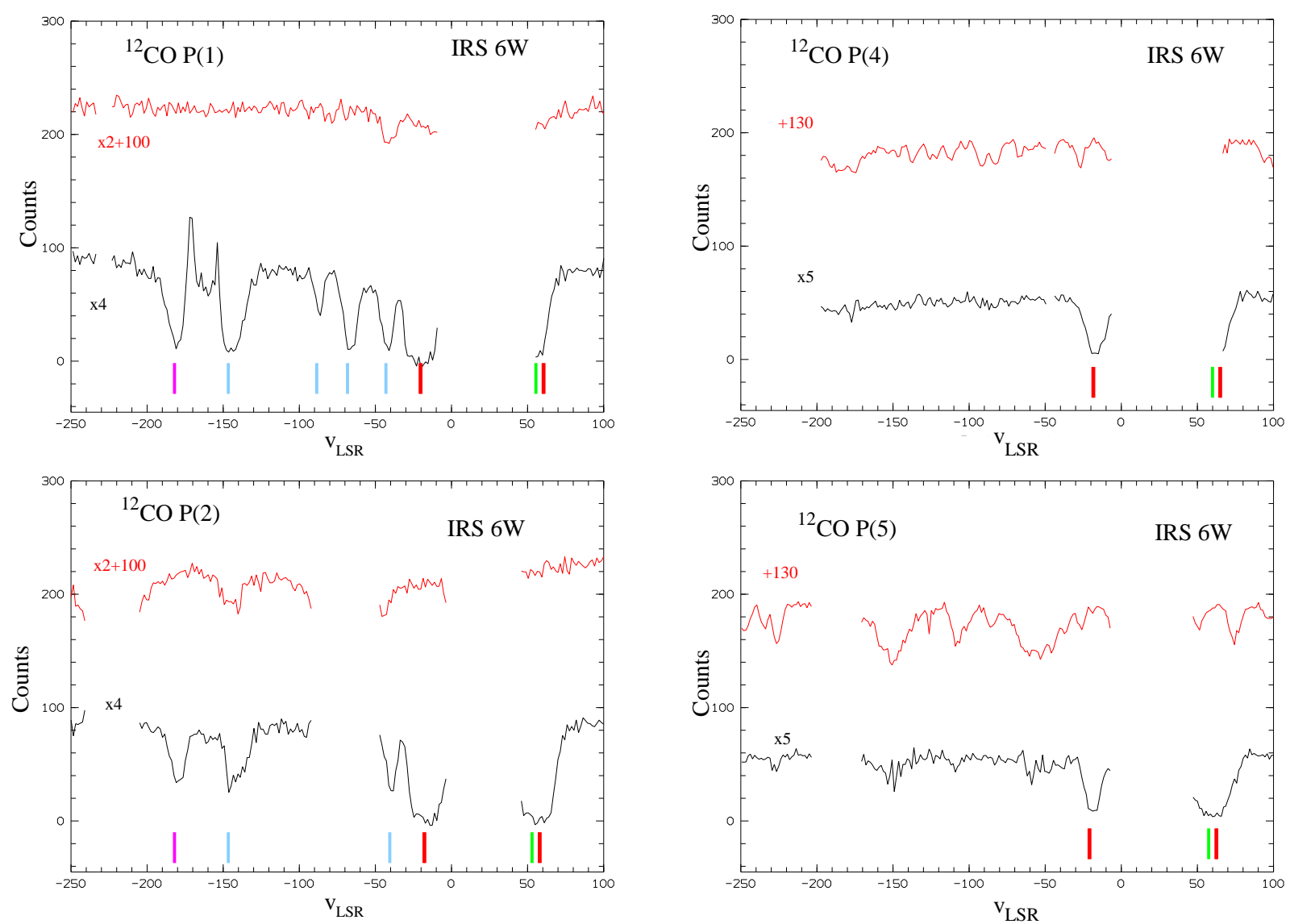

Fig. A.6. Spectra (in black) of IRS $6 \mathrm{~W}$ in the ${ }^{12} \mathrm{CO} \mathrm{P}(1), \mathrm{P}(2), \mathrm{P}(4)$ and $\mathrm{P}(5)$ transitions. Also shown (in red) are the spectra of the standard stars observed close to the science observation to correct for telluric lines. The blanked areas correspond to regions in the spectrum where the telluric corrections were not successful. The red arrows indicate the local absorptions at velocities listed in Table 1 . The other colored arrows indicate the absorptions at the same velocities as in Figs. 3 and 4. The scaling and/or shifting factors of the spectra are given in red and black for the standard star and the GC object, respectively. 
J. Moultaka et al.: CRIRES observations of the GC
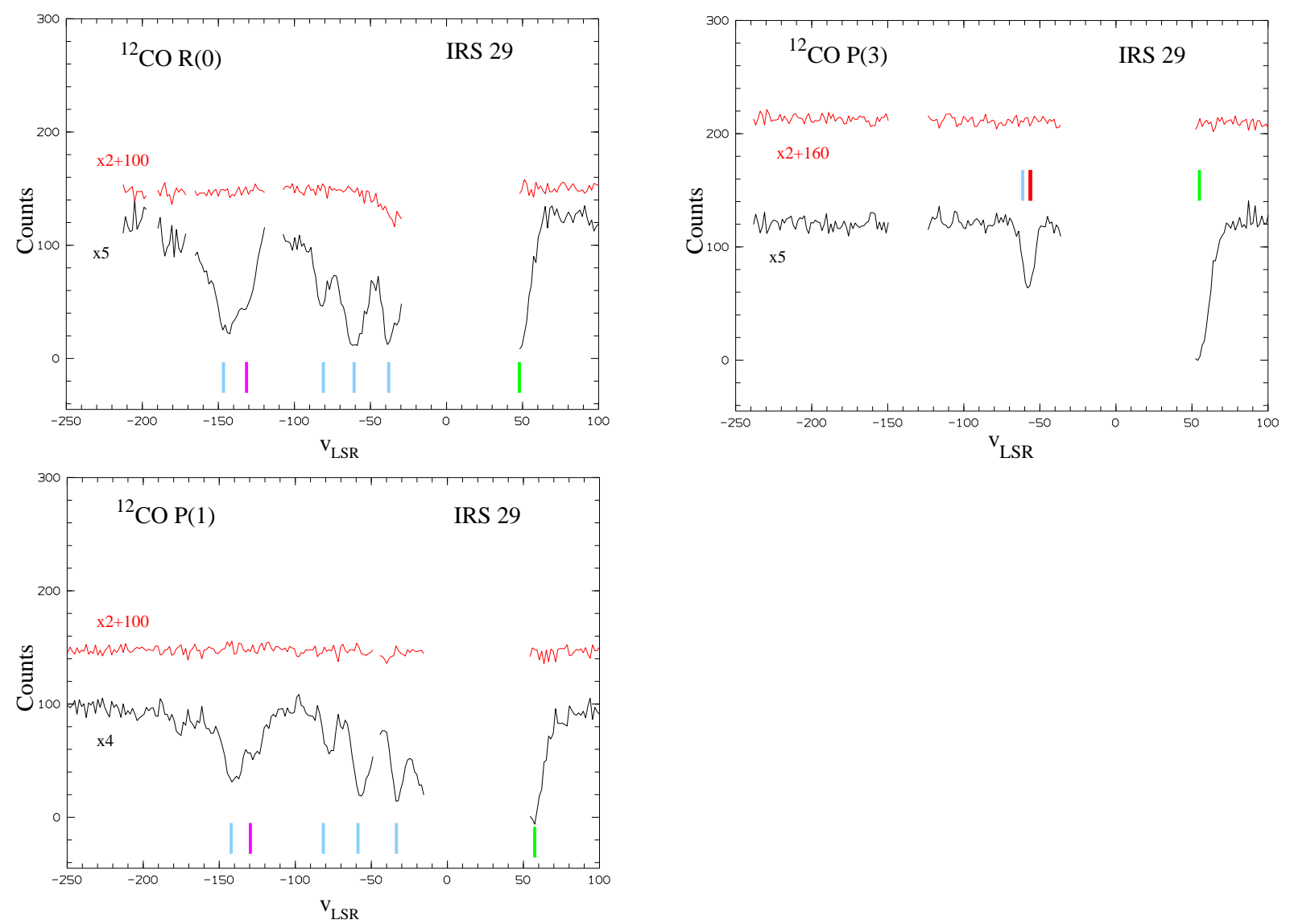

Fig. A.7. Spectra (in black) of IRS 29 in the ${ }^{12} \mathrm{CO} R(0), \mathrm{P}(1)$ and $\mathrm{P}(3)$ transitions. Also shown (in red) are the spectra of the standard stars observed close to the science observation to correct for telluric lines. The blanked areas correspond to regions in the spectrum where the telluric corrections were not successful. The red arrows indicate the local absorptions at velocities listed in Table 1 . The other colored arrows indicate the absorptions at the same velocities as in Figs. 3 and 4. The scaling and/or shifting factors of the spectra are given in red and black for the standard star and the GC object, respectively. 

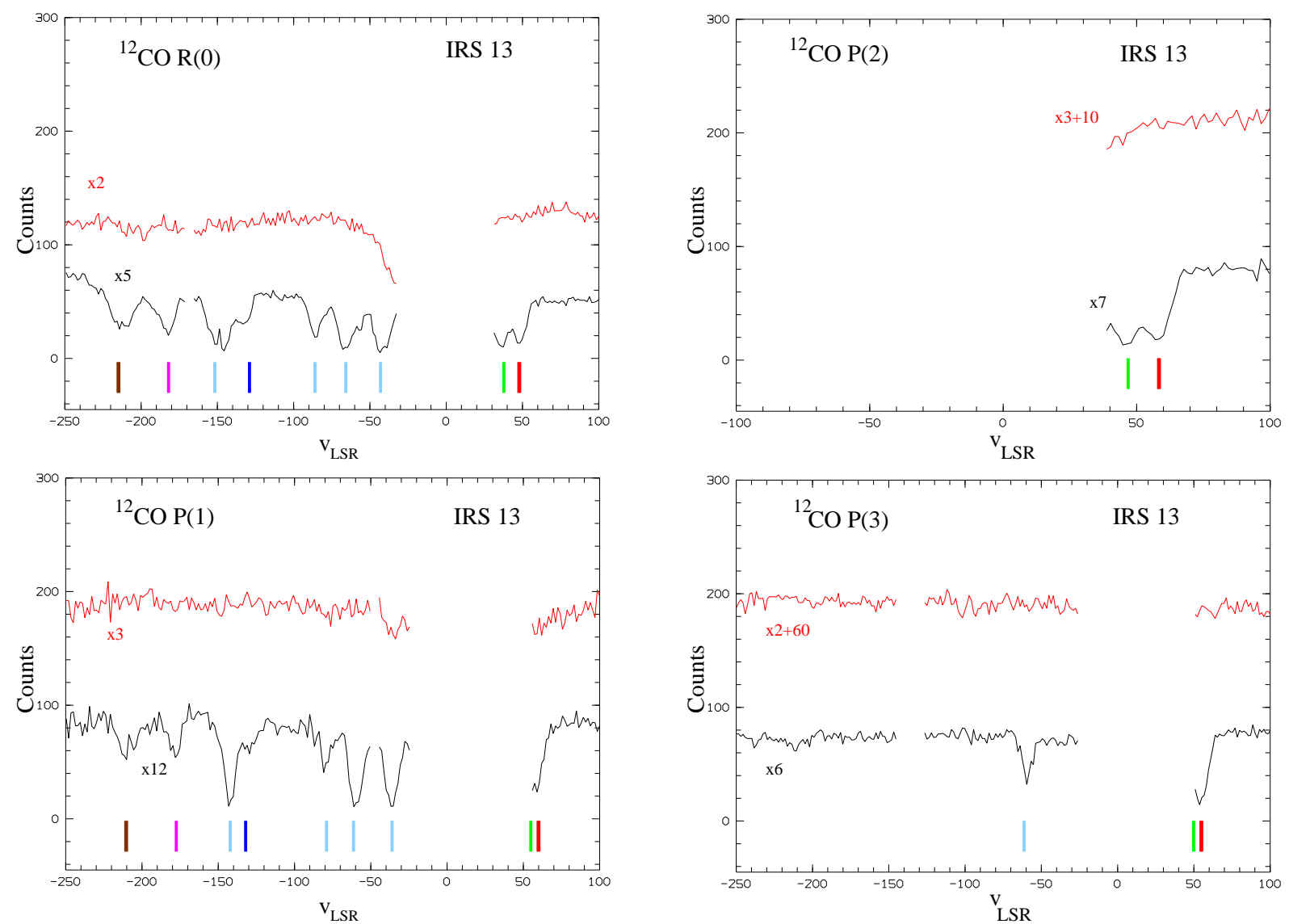

Fig. A.8. Spectra (in black) of IRS 13 in the ${ }^{12} \mathrm{CO} R(0), \mathrm{P}(1), \mathrm{P}(2)$ and $\mathrm{P}(3)$ transitions. Also shown (in red) are the spectra of the standard stars observed close to the science observation to correct for telluric lines. The blanked areas correspond to regions in the spectrum where the telluric corrections were not successful. The red arrows indicate the local absorptions at velocities listed in Table 1 . The other colored arrows indicate the absorptions at the same velocities as in Figs. 3 and 4. Finally, the brown arrows indicate the line at $-210 \mathrm{~km} \mathrm{~s}^{-1}$. The scaling and/or shifting factors of the spectra are given in red and black for the standard star and the GC object, respectively. 
J. Moultaka et al.: CRIRES observations of the GC
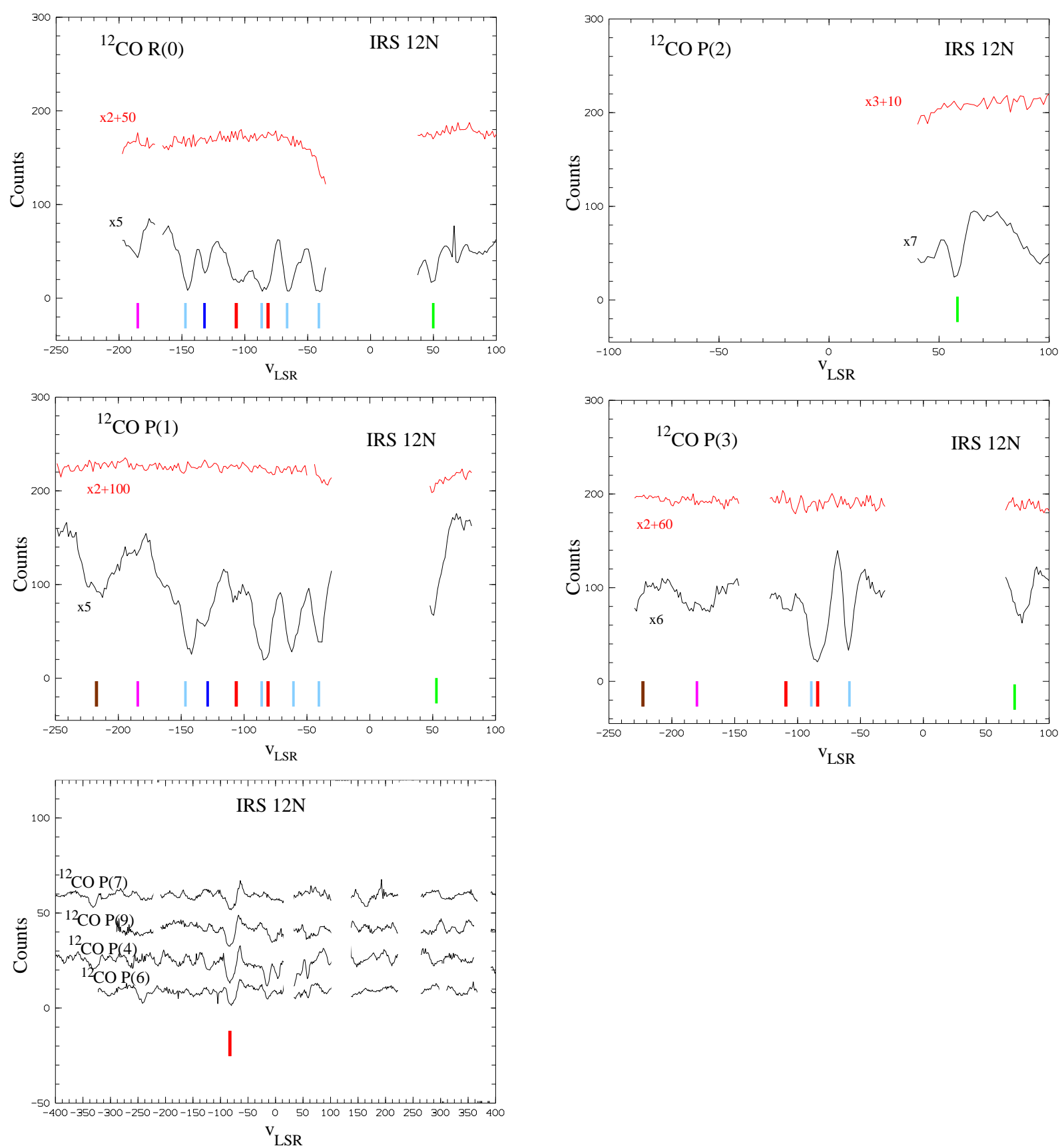

Fig. A.9. Spectra (in black) of IRS $12 \mathrm{~N}$ in the ${ }^{12} \mathrm{CO} R(0), \mathrm{P}(1), \mathrm{P}(2), \mathrm{P}(3), \mathrm{P}(4), \mathrm{P}(6), \mathrm{P}(7)$ and $\mathrm{P}(9)$ transitions. Also shown (in red) are the spectra of the standard stars observed close to the science observation to correct for telluric lines. The blanked areas correspond to regions in the spectrum where the telluric corrections were not successful. The red arrows indicate the local absorptions at velocities listed in Table 1 . The other colored arrows indicate the absorptions at the same velocities as in Figs. 3 and 4. Finally, the brown arrows indicate the line at $-210 \mathrm{~km} \mathrm{~s}^{-1}$. The scaling and/or shifting factors of the spectra are given in red and black for the standard star and the GC object, respectively. 

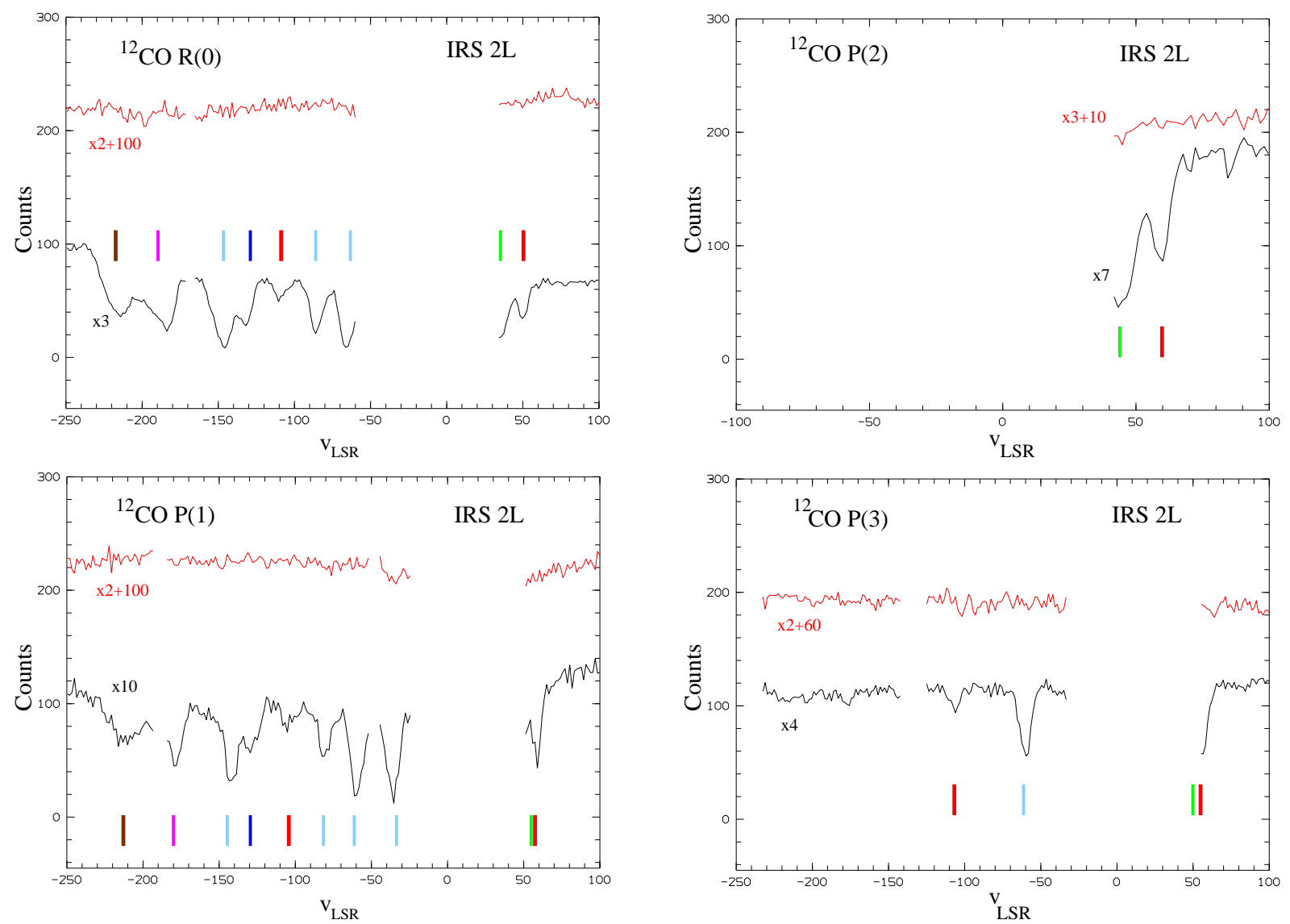

Fig. A.10. Spectra (in black) of IRS $2 \mathrm{~L}$ in the ${ }^{12} \mathrm{CO} R(0), \mathrm{P}(1), \mathrm{P}(2)$ and $\mathrm{P}(3)$ transitions. Also shown (in red) are the spectra of the standard stars observed close to the science observation to correct for telluric lines. The blanked areas correspond to regions in the spectrum where the telluric corrections were not successful. The red arrows indicate the local absorptions at velocities listed in Table 1 . The other colored arrows indicate the absorptions at the same velocities as in Figs. 3 and 4. Finally, the brown arrows indicate the line at $-210 \mathrm{~km} \mathrm{~s}^{-1}$. The scaling and/or shifting factors of the spectra are given in red and black for the standard star and the GC object, respectively. 
J. Moultaka et al.: CRIRES observations of the GC
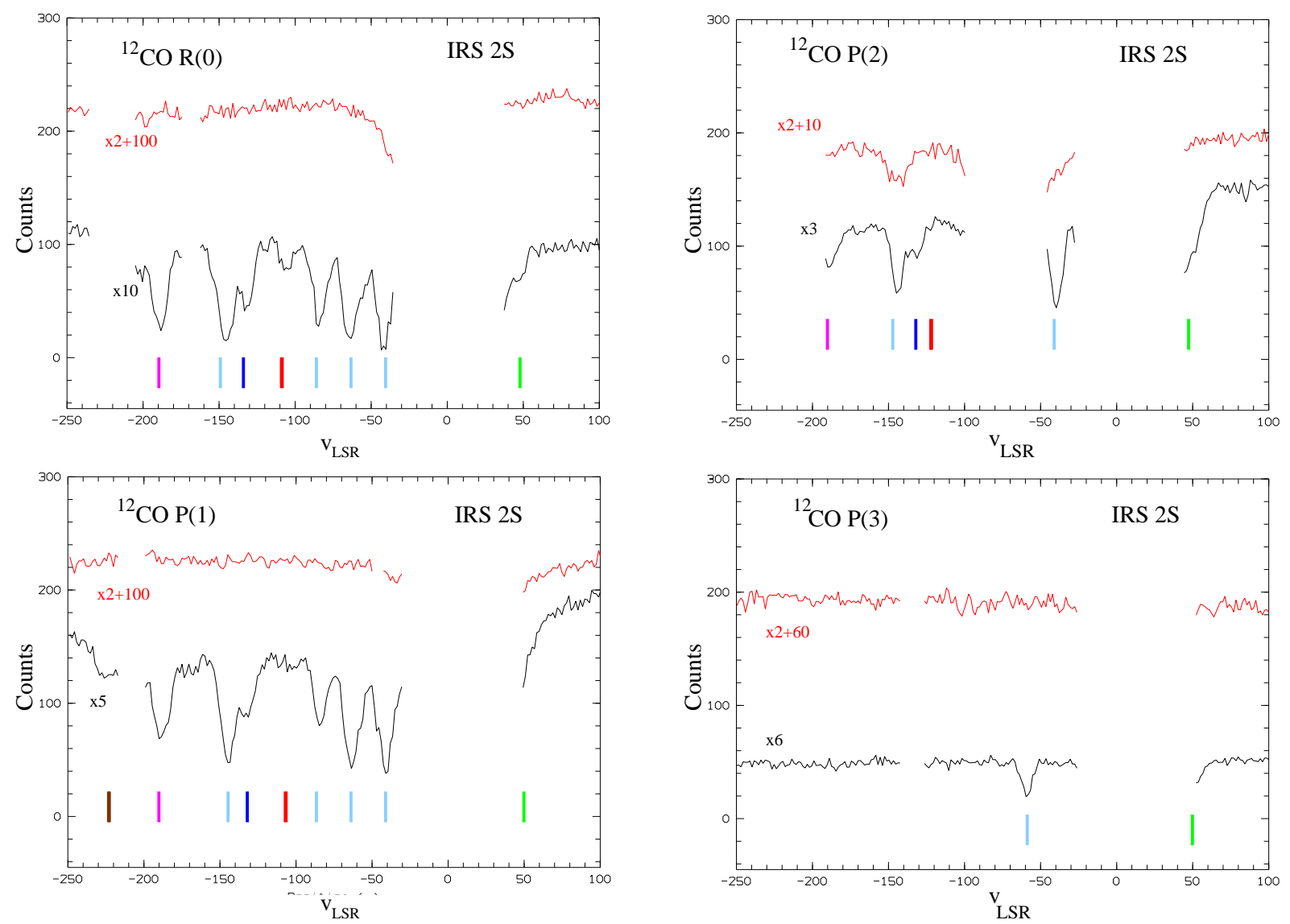

Fig. A.11. Spectra (in black) of IRS $2 \mathrm{~S}$ in the ${ }^{12} \mathrm{CO} \mathrm{R}(0), \mathrm{P}(1), \mathrm{P}(2)$ and $\mathrm{P}(3)$ transitions. Also shown (in red) are the spectra of the standard stars observed close to the science observation to correct for telluric lines. The blanked areas correspond to regions in the spectrum where the telluric corrections were not successful. The red arrows indicate the local absorptions at velocities listed in Table 1 . The other colored arrows indicate the absorptions at the same velocities as in Figs. 3 and 4. Finally, the brown arrow indicates the line at $-210 \mathrm{~km} \mathrm{~s}^{-1}$. The scaling and/or shifting factors of the spectra are given in red and black for the standard star and the GC object, respectively. 
A\&A 626, A44 (2019)
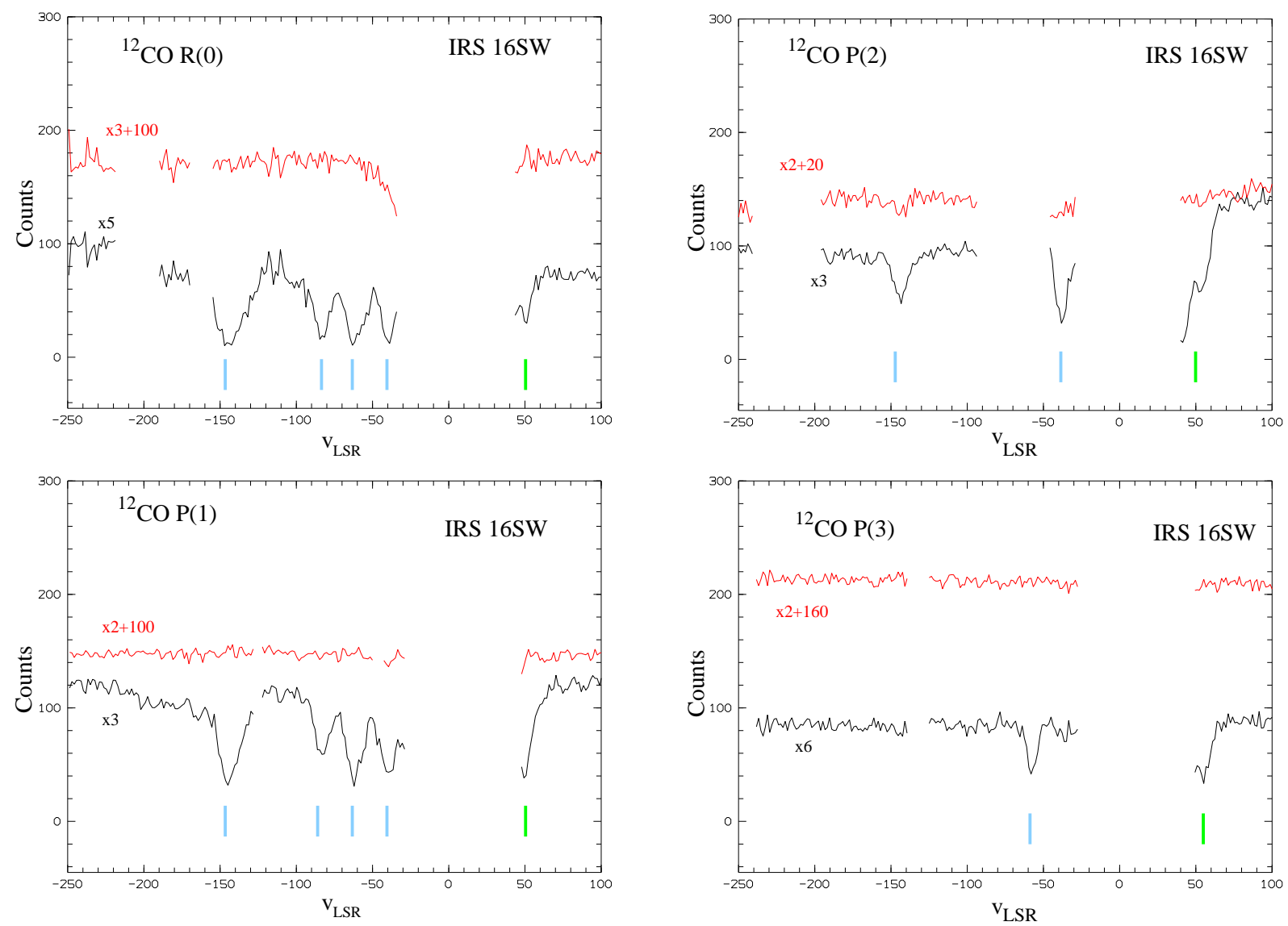

Fig. A.12. Spectra (in black) of IRS $16 \mathrm{SW}$ in the ${ }^{12} \mathrm{CO} R(0), \mathrm{P}(1), \mathrm{P}(2)$ and $\mathrm{P}(3)$ transitions. Also shown (in red) are the spectra of the standard stars observed close to the science observation to correct for telluric lines. The blanked areas correspond to regions in the spectrum where the telluric corrections were not successful. The red arrows indicate the local absorptions at velocities listed in Table 1 . The other colored arrows indicate the absorptions at the same velocities as in Figs. 3 and 4. The scaling and/or shifting factors of the spectra are given in red and black for the standard star and the GC object, respectively. 
J. Moultaka et al.: CRIRES observations of the GC
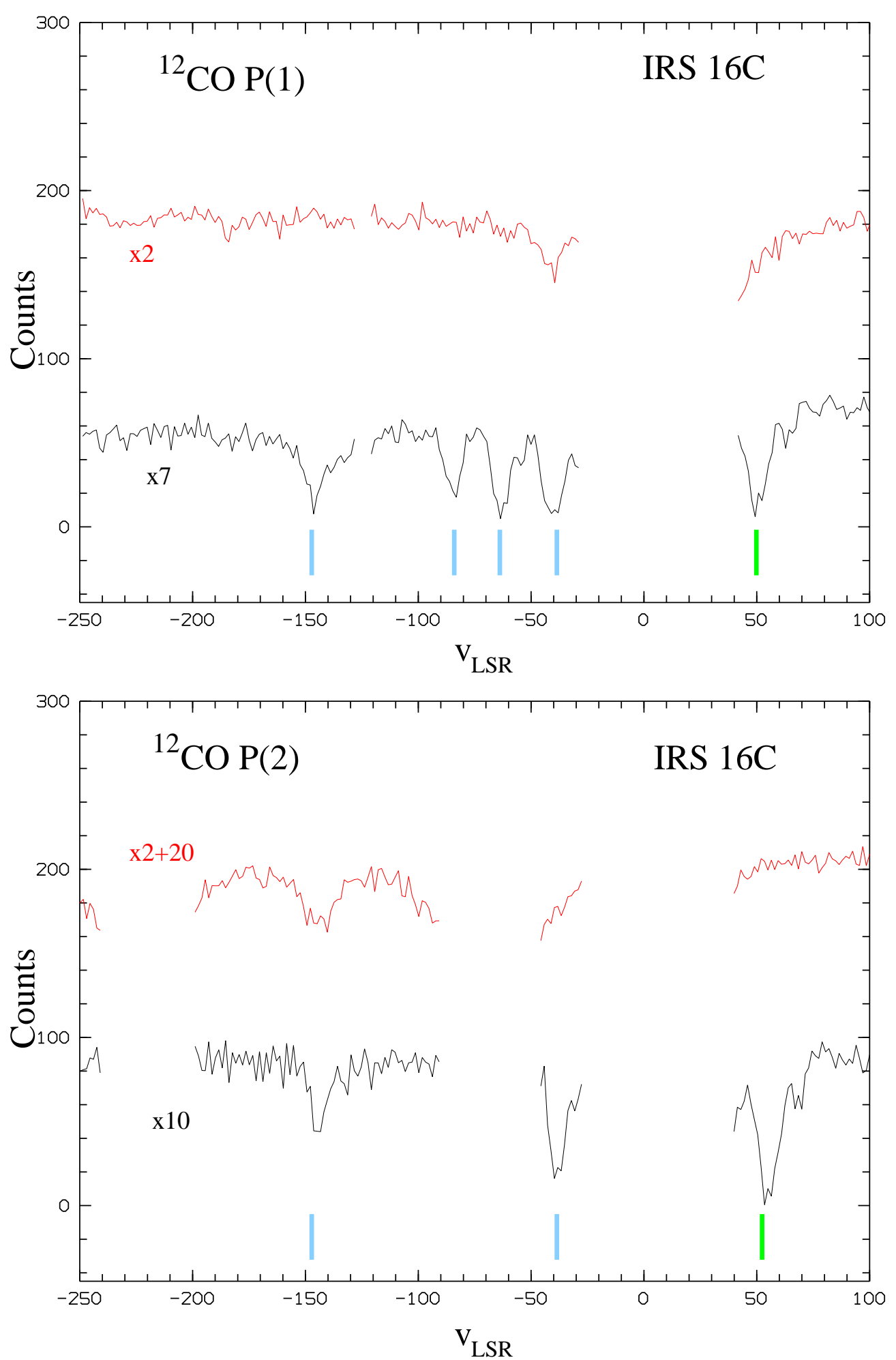

Fig. A.13. Spectra (in black) of IRS $16 \mathrm{C}$ in the ${ }^{12} \mathrm{CO} \mathrm{P}(1)$ and $\mathrm{P}(2)$ transitions. Also shown (in red) are the spectra of the standard stars observed close to the science observation to correct for telluric lines. The blanked areas correspond to regions in the spectrum where the telluric corrections were not successful. The red arrows indicate the local absorptions at velocities listed in Table 1 . The other colored arrows indicate the absorptions at the same velocities as in Figs. 3 and 4. The scaling and/or shifting factors of the spectra are given in red and black for the standard star and the GC object, respectively. 